\title{
Muon-electron scattering at NLO
}

\author{
Massimo Alacevich, ${ }^{a, b}$ Carlo M. Carloni Calame, ${ }^{b}$ Mauro Chiesa, ${ }^{c}$ Guido Montagna, ${ }^{a, b}$ \\ Oreste Nicrosini ${ }^{b}$ and Fulvio Piccinini ${ }^{b}$ \\ ${ }^{a}$ Dipartimento di Fisica, Università di Pavia, \\ Via A. Bassi 6, Pavia 27100, Italy \\ ${ }^{b}$ INFN, Sezione di Pavia, \\ Via A. Bassi 6, Pavia 27100, Italy \\ ${ }^{c}$ Institut für Theoretische Physik und Astrophysik, Julius-Maximilians-Universität Würzburg, \\ Emil-Hilb-Weg 22, Würzburg D-97074, Germany \\ E-mail: massimo.alacevich@pv.infn.it, \\ carlo.carloni.calame@pv.infn.it, \\ mauro.chiesa@physik.uni-wuerzburg.de, guido.montagna@pv.infn.it, \\ oreste.nicrosini@pv.infn.it, fulvio.piccinini@pv.infn.it
}

ABSTRACT: We consider the process of muon-electron elastic scattering, which has been proposed as an ideal framework to measure the running of the electromagnetic coupling constant at space-like momenta and determine the leading-order hadronic contribution to the muon $g-2$ (MUonE experiment). We compute the next-to-leading (NLO) contributions due to QED and purely weak corrections and implement them into a fully differential Monte Carlo event generator, which is available for first experimental studies. We show representative phenomenological results of interest for the MUonE experiment and examine in detail the impact of the various sources of radiative corrections under different selection criteria, in order to study the dependence of the NLO contributions on the applied cuts. The study represents the first step towards the realisation of a high-precision Monte Carlo code necessary for data analysis.

KEYwORDS: NLO Computations

ArXiv EPrint: 1811.06743 


\section{Contents}

1 Introduction 1

2 Details of the NLO calculation 3

2.1 NLO QED corrections 3

2.2 NLO electroweak corrections 5

2.3 QED corrections in the vanishing electron mass limit 5

3 Numerical results $\quad 6$

3.1 Integrated cross sections $\quad 8$

$\begin{array}{lll}3.2 & \text { Differential cross sections } & 11\end{array}$

4 Conclusions $\quad 19$

\section{Introduction}

The anomalous magnetic moment of the muon $a_{\mu}=(g-2)_{\mu} / 2$ represents a formidable test of the Standard Model (SM) of particle physics $[1,2]$. There is a persisting tension between the measured value, as provided by the BNL-E821 experiment with an accuracy of $0.54 \mathrm{ppm}$ [3], and the SM prediction, which presently exceeds the $3 \sigma$ level [4-7]. This discrepancy could be ascribed to some uncontrolled experimental or theoretical systematics or could signal the presence of New Physics beyond the SM. Two next-generation muon $g-2$ experiments at Fermilab [8] and J-PARC [9] plan to improve the experimental error by a factor of four, which constitutes a challenge for the theory and has triggered a lot of new theoretical efforts $[4,5]$.

The calculation of $a_{\mu}$ in the SM requires the evaluation of subtle quantum loop effects and its present uncertainty, at the level of $3.5 \times 10^{-10}$, is dominated by strong interaction contributions, which can not be computed perturbatively at low energies. The main sources of error come from the evaluation of the leading-order hadronic correction, $a_{\mu}^{\mathrm{HLO}}$, and of the hadronic light-by-light contribution. In particular, the most precise estimates of $a_{\mu}^{\mathrm{HLO}}$ rely on the calculation of a dispersion integral of the hadron production cross section measured in $e^{+} e^{-}$annihilation at a few $\mathrm{GeV}$ scale. This data-driven determination of the $a_{\mu}^{\mathrm{HLO}}$ contribution to the $g-2$ is affected by uncertainties coming from the experimental error, as well as from delicate issues in the treatment and combination of different data corresponding to many exclusive channels $[4,6,7]$. Hence, alternative and independent approaches to the standard evaluation of $a_{\mu}^{\mathrm{HLO}}$ via time-like data are of utmost importance to shed light on the situation. ${ }^{1}$

\footnotetext{
${ }^{1}$ Recent progress in lattice QCD calculations of $a_{\mu}^{\mathrm{HLO}}$ is documented in refs. [10-14].
} 
In this respect, it has been proposed in recent times [15] to derive the leading-order hadronic contribution to the muon anomaly from a measurement of the effective electromagnetic coupling in the space-like region (and in particular of its hadronic contribution $\left.\Delta \alpha_{\text {had }}\left(q^{2}\right)\right)$, following ideas first put forward in ref. [16]. Shortly afterwards, the process of muon-electron elastic scattering, i.e. $\mu e \rightarrow \mu e$, as measurable by scattering high-energy muons on electrons at rest, has been recognised as an ideal process to perform such a measurement [17]. ${ }^{2}$ An experiment devoted to the measurement of $\alpha_{\mathrm{QED}}(t)$, where $t$ is the space-like squared momentum transfer, via $\mu e$ scattering (MUonE) is presently under consideration at CERN in the context of the Physics Beyond Colliders study group. ${ }^{3}$ The idea consists in performing a fixed-target experiment, by using a $150 \mathrm{GeV}$ high-intensity muon beam, presently available at the CERN North area, incident on atomic electrons of a low- $Z$ target.

The challenge of the proposed experiment is the feasibility of achieving a statistical and systematic uncertainty in the measurement of the $\mu e$ differential cross sections at the level of $10 \mathrm{ppm}$. If this task is accomplished, the new space-like determination of the leading hadronic contribution to the muon $g-2$ will turn out to be competitive with the present time-like approach. In addition to the systematics of experimental nature, the calculation of the radiative corrections to the $\mu e$ cross section represents a further source of systematic uncertainty to be taken carefully under control. Ultimately, this calculation will be necessary according to the highest standards of theoretical precision, in order to cope with the very demanding requirement of accuracy.

In this paper, we take a first step towards the calculation of higher-order contributions to $\mu e \rightarrow \mu e$ scattering, by computing the full set of NLO corrections to the process in the $\mathrm{SM}$ as due to QED and purely weak effects. We calculate the above corrections without any approximation, i.e. retaining finite lepton (muon and electron) mass contributions, and make them available in the form of a fully flexible Monte Carlo (MC) code, which is unavoidable to analyse the precision data of the MUonE experiment. We also scrutinise the size of the various sources of radiative corrections, in order to provide a guideline to future efforts in the calculation of NNLO corrections and resummation.

The NLO QED corrections to the $\mu e$ scattering cross section were computed long time ago [20-26] and recently revisited in ref. [27]. However, the above calculations contain some assumptions (vanishing electron mass, soft photon approximation), which do not apply to the present work. To the best of our knowledge, one-loop weak corrections, due to the presence of electroweak bosons in the internal loops, have never been considered so far. Just the contribution of the $\gamma-Z$ interference at the tree-level was computed in ref. [24] and compared to $O(\alpha)$ QED corrections at relatively high energies.

On the NNLO side, the master integrals for the two-loop planar and non-planar box diagrams in QED have been evaluated in ref. [28] and ref. [29], respectively, retaining full dependence on the muon mass. NNLO hadronic corrections to $\mu$ e scattering, given by diagrams with a hadronic vacuum polarisation insertion in the photon propagator, have

\footnotetext{
${ }^{2} \mathrm{~A}$ method to measure the running of the QED coupling in the space-like region using small-angle Bhabha scattering was proposed in ref. [18] and applied to LEP data by OPAL collaboration [19].

${ }^{3}$ See https://indico.cern.ch/event/644287/.
} 
been computed recently in refs. [30, 31]. Further theoretical progress in the calculation of NNLO corrections and resummation was presented at the MITP topical workshop "The evaluation of the leading hadronic contribution to the muon anomalous magnetic moment", held in Mainz in February 2018. ${ }^{4}$

The paper is organised as follows. In section 2 we describe the details of the NLO calculation. In section 3 we present and discuss our phenomenological results, both at the level of integrated cross sections (section 3.1) and differential observables (section 3.2). We study in particular the impact of the NLO corrections as a function of the imposed event selection criteria and scrutinise the rôle played by the different gauge-invariant subsets of corrections. In section 4 we draw our conclusions and discuss the perspectives of our work.

\section{Details of the NLO calculation}

In the present section, we describe the details of our theoretical approach. We first discuss the computation of the NLO QED and electroweak contributions without any approximation, i.e. retaining full dependence on the lepton masses. Next, we mention the procedure followed for the calculation of the QED corrections in the limit of vanishing electron mass, which is sketched in section 3 .

\subsection{NLO QED corrections}

The differential unpolarised cross section of muon-electron elastic scattering

$$
\mu^{ \pm}\left(p_{1}\right)+e^{-}\left(p_{2}\right) \rightarrow \mu^{ \pm}\left(p_{3}\right)+e^{-}\left(p_{4}\right)
$$

at LO in QED is given by

$$
\frac{d \sigma}{d t}=\frac{1}{\lambda\left(s, m_{\mu}^{2}, m_{e}^{2}\right)} \frac{4 \pi \alpha^{2}}{t^{2}}\left[\left(s-m_{\mu}^{2}-m_{e}^{2}\right)^{2}+s t+\frac{1}{2} t^{2}\right] .
$$

In eq. (2.1) $\alpha$ is the fine structure constant, $\lambda$ is the Källén function and $s, t$ are the usual Mandelstam variables, which are calculated with massive four momenta of the incoming and outgoing muon $\left(p_{1}\right.$ and $\left.p_{3}\right)$ and of the incoming and outgoing electron $\left(p_{2}\right.$ and $\left.p_{4}\right)$. In our calculation, we consider the scattering initiated by both positive and negative muons, both options being possible in the muon beam available at the CERN North Area.

In the electroweak theory, the NLO corrections to the process consist of QED and purely weak contributions. Since $\mu$ e scattering is a neutral-current process, the two subsets are separately gauge invariant and can be treated separately. A priori, the QED corrections are expected to be the dominant contribution, as photons which are emitted collinear to a lepton give rise to enhanced logarithmic corrections of the form $\alpha \log \left(Q^{2} / m_{\ell}^{2}\right)$ in the large $Q^{2}$ limit, where $m_{\ell}$ is the lepton mass and $Q$ some typical energy scale.

We calculate the $2 \rightarrow 2$ amplitude including one-loop virtual corrections in the onshell renormalization scheme, whereas real photon corrections are induced by the $2 \rightarrow 3$ bremsstrahlung process $\mu e \rightarrow \mu e+\gamma$. The latter contribution is infrared (IR) divergent,

\footnotetext{
${ }^{4}$ See the material available at the link https://indico.mitp.uni-mainz.de/event/128/.
} 
but its sum with vertex and box corrections is IR-finite. We regularise the IR singularities according to the standard QED procedure of assigning a vanishingly small mass to the photon in the computation of the virtual and real contributions. Ultraviolet (UV) divergences associated to loop diagrams are treated using dimensional regularisation.

In formulae, the NLO cross section is split into two contributions and computed as follows

$$
\sigma_{\mathrm{NLO}}=\sigma_{\mu e \rightarrow \mu e}+\sigma_{\mu e \rightarrow \mu e \gamma} \equiv \sigma_{2 \rightarrow 2}+\sigma_{2 \rightarrow 3} .
$$

In eq. (2.2), $\sigma_{2 \rightarrow 2}$ is given by the sum of the LO cross section and the NLO one containing virtual photonic corrections and reads explicitly as follows

$$
\sigma_{2 \rightarrow 2}=\sigma_{\mathrm{LO}}+\sigma_{\mathrm{NLO}}^{\text {virtual }}=\frac{1}{F} \int d \Phi_{2}\left(\left|\mathcal{A}_{\mathrm{LO}}\right|^{2}+2 \operatorname{Re}\left[\mathcal{A}_{\mathrm{LO}}^{\dagger} \times \mathcal{A}_{\mathrm{NLO}}^{\text {virtual }}(\lambda)\right]\right)
$$

where $F=2 \sqrt{\left(s-m_{e}^{2}-m_{\mu}^{2}\right)^{2}-4 m_{e}^{2} m_{\mu}^{2}}$ is the incoming flux factor, $d \Phi_{2}$ the $2 \rightarrow 2$ phase space volume and $\lambda$ the fictitious photon mass.

For the calculation of the $2 \rightarrow 3$ real photon contribution, we introduce a phase space slicing in terms of an arbitrarily small cutoff on the photon energy $\omega$ and compute $\sigma_{2 \rightarrow 3}$ according to the following formula

$$
\sigma_{2 \rightarrow 3}=\frac{1}{F} \int_{\omega>\lambda} d \Phi_{3}\left|\mathcal{A}_{\mathrm{NLO}}^{1 \gamma}\right|^{2}=\frac{1}{F}\left(\int_{\lambda<\omega<\omega_{s}} d \Phi_{3}\left|\mathcal{A}_{\mathrm{NLO}}^{1 \gamma}\right|^{2}+\int_{\omega>\omega_{s}} d \Phi_{3}\left|\mathcal{A}_{\mathrm{NLO}}^{1 \gamma}\right|^{2}\right)
$$

where $\omega_{s}$ is a soft-hard slicing separator, with $\lambda \ll \omega_{s} \ll \sqrt{s}$, and $d \Phi_{3}$ is the $2 \rightarrow 3$ phase space element. The first contribution in the r.h.s. of eq. (2.4) is calculated retaining the photon mass $\lambda$, while in the second one the photon is treated as massless. By integrating the first contribution analytically, eq. (2.4) can be recast as follows

$$
\sigma_{2 \rightarrow 3}=\Delta_{s}\left(\lambda, \omega_{s}\right) \int d \sigma_{\mathrm{LO}}+\frac{1}{F} \int_{\omega>\omega_{s}} d \Phi_{3}\left|\mathcal{A}_{\mathrm{NLO}}^{1 \gamma}\right|^{2}
$$

where $\Delta_{s}\left(\lambda, \omega_{s}\right)$ is the eikonal factor for the real radiation correction in the soft photon approximation, whose expression can be found for instance in ref. [32].

The contribution due to vacuum polarisation is taken into account using the effective charge as an overall factor through the replacement $\alpha \rightarrow \alpha(t)$ in the LO and NLO cross section. The running of the QED coupling is calculated using the following Dysonresummation expression

$$
\alpha(t)=\frac{\alpha}{1-\Delta \alpha(t)}
$$

where

$$
\Delta \alpha(t)=\Delta \alpha_{\text {lep }}(t)+\Delta \alpha_{\text {top }}(t)+\Delta \alpha_{\text {had }}(t) .
$$

In this work, we implement the leptonic correction $\Delta \alpha_{\text {lep }}$ and the (tiny) top-quark contribution $\Delta \alpha_{\text {top }}$ in one-loop approximation ${ }^{5}$ and the hadronic correction is taken into account

\footnotetext{
${ }^{5}$ Note however that the leptonic contribution is known at three [33] and at four loops [34] in QED: the implementation in the present calculation of higher-order corrections is straightforward and their impact is studied in section 3.2. We also refrain from including any weak correction beyond one-loop approximation (see e.g. [35]), because they are found to be negligible already at NLO, as discussed in section 3.2.
} 
according to the dispersive approach implemented in the latest version of the hadr5n16.f routine [36]. ${ }^{6}$ Note, however, that we use the value $\alpha=\alpha(0)$ of the fine structure constant for the coupling of the photon to the external charged legs. We would like also to emphasize that we use here the hadr5n16.f routine only to get the expected effect on the running of $\alpha$ induced by $\Delta \alpha_{\text {had }}(t)$, which will be extracted from MUonE data by inserting eq. (2.7) into eq. (2.6) and solving for $\Delta \alpha_{\text {had }}(t)$.

Concerning the method of calculation and related cross-checks, all the Feynman diagrams for virtual and real photon contributions were manipulated with the help of the symbolic manipulation program ForM $[37,38]$, retaining full dependence on fermion masses and helicities. The evaluation of one-loop tensor coefficients and scalar $2 / 3 / 4$ points functions was performed using the package LoopTools $[39,40]$ and cross-checked against the ColLIER software [41], finding perfect agreement between the results of the two libraries. UV-finiteness and independence of the $\lambda$ parameter have been checked analytically while the independence on $\omega_{s}$ has been verified with high numerical accuracy in the range $10^{-7} \mathrm{MeV}<\omega_{s}<10^{-3} \mathrm{MeV}$.

\subsection{NLO electroweak corrections}

The calculation of the full set of NLO corrections in the electroweak theory has been performed according to the following details: the tree-level and one-loop matrix elements for the virtual and real corrections have been evaluated without any approximation with the computer program RECOLA [42], which internally uses the CoLLIER library for the oneloop scalar [43-46] and tensor integrals [47-49]. In accordance with the calculation of the QED corrections, soft-photon singularities have been regularised in terms of an infinitesimal photon mass parameter and renormalization has been performed in the on-shell scheme. In order to compare consistently with the results of the QED calculation at the level of purely photonic corrections, the fermionic one-loop contributions to the photon propagator, which are automatically computed by the RECOLA program, have been calculated analytically and subtracted from the RECOLA output.

\subsection{QED corrections in the vanishing electron mass limit}

The results for the NLO corrections discussed above include the contributions due to finite lepton mass effects, such as corrections of the kind $\alpha m_{\ell}^{2} / Q^{2} \log \left(Q^{2} / m_{\ell}^{2}\right)$ and $\alpha m_{\ell}^{2} / Q^{2}$. As a consequence of the available c.m. energy and typical values of the squared momentum transfer relevant for the MUonE experiment, muon mass effects can not be neglected and contribute, together with logarithmic enhanced terms, to make the whole NLO muon correction. However, also because of the complexity of a two-loop calculation in the presence of two different massive fermions $[28,29,50]$, it is worth assessing the rôle played by the contributions induced by a finite electron mass within the one-loop calculation.

To this end, we performed a calculation of the NLO QED corrections by treating the electron as massless, wherever possible. The adopted approximation is detailed in section 3 .

\footnotetext{
${ }^{6}$ Available at http://www-com.physik.hu-berlin.de/ fjeger/software.html.
} 


\section{Numerical results}

In this section, we show and discuss the phenomenological results obtained by using a fully differential MC code which implements the theoretical approach described in section 2. For definiteness, we remark that all calculations and simulations are performed in the center of mass (c.m.) frame and then the momenta are boosted to the laboratory frame, where the initial-state electron is at rest.

We present numerical results both for the $\mu^{-} e^{-} \rightarrow \mu^{-} e^{-}$and $\mu^{+} e^{-} \rightarrow \mu^{+} e^{-}$process, since both options are relevant for the MUonE experiment and the radiative corrections differ in the two cases, as it will be shown in the following.

For the QED calculation, the input parameters are set to:

$$
\begin{array}{rlrl}
\alpha(0) & \equiv \alpha=1 / 137.03599907430637 & \\
m_{e} & =0.510998928 \mathrm{MeV} & m_{\mu} & =105.6583715 \mathrm{MeV} \\
m_{\tau} & =1.77682 \mathrm{GeV} & m_{\text {top }} & =175.6 \mathrm{GeV} .
\end{array}
$$

The electroweak predictions are obtained in a scheme where $\alpha, M_{W}$ and $M_{Z}$ are input parameters. Besides the inputs of eq. (3.1), NLO electroweak predictions provided by RECOLA further require $M_{Z}=91.1876 \mathrm{GeV}, M_{W}=80.376 \mathrm{GeV}, M_{H}=125 \mathrm{GeV}, \Gamma_{Z}=$ $\Gamma_{W}=0 \mathrm{GeV}$ and the masses of the light quarks. The latter are needed for the calculation of the fermionic one-loop corrections to the photon and $Z$ propagators. As remarked above, the fermionic one-loop corrections to the photon propagator are analytically subtracted, leaving a tiny dependence of the full NLO electroweak result on the light quark masses in the $Z$ self energy and $\gamma / Z$ mixing (which is anyway not singular as the quark masses go to zero). Their value is set in such a way to reproduce $\Delta \alpha_{\text {had }}^{(5)}\left(M_{Z}^{2}\right)$ (see for instance [51]).

For the energy of the incoming muons, we assume $E_{\mu}^{\text {beam }}=150 \mathrm{GeV}$, which is the energy of the M2 beam line of the CERN SPS. Note that, under the fixed-target configuration of the MUonE experiment, the c.m. energy corresponding to this muon energy is given by $\sqrt{s} \simeq 0.405541 \mathrm{GeV}$ and that the Lorentz $\gamma$ factor boosting the c.m. frame into the lab frame is $\gamma \simeq 370$. We stress that in the lab frame the relation $t_{e e} \equiv\left(p_{2}-p_{4}\right)^{2}=2 m_{e}^{2}-2 m_{e} E_{e}$ (where $E_{e}$ is the energy of the scattered electron) holds, which implies that a lower limit on $E_{e}$ corresponds to an upper limit on $t_{e e}$.

In this kinematical condition, the collinear logarithms $L_{e}=\ln \left(s / m_{e}^{2}\right)$ and $L_{\mu}=$ $\ln \left(s / m_{\mu}^{2}\right)$ are of the order of $L_{e} \simeq 13.4$ and $L_{\mu} \simeq 2.7$, respectively.

In order to study the dependence of the radiative corrections on the applied cuts, we consider four different event selections defined by the following criteria:

1. $\theta_{e}, \theta_{\mu}<100 \mathrm{mrad}$ and $E_{e}>0.2 \mathrm{GeV}$ (i.e. $t_{e e} \lesssim-2.04 \cdot 10^{-4} \mathrm{GeV}^{2}$ ). The angular cuts model the typical acceptance conditions of the experiment and the electron energy threshold is imposed to guarantee the presence of two charged tracks in the detector;

2. $\theta_{e}, \theta_{\mu}<100 \mathrm{mrad}$ and $E_{e}>1 \mathrm{GeV}$ (i.e. $t_{e e} \lesssim-1.02 \cdot 10^{-3} \mathrm{GeV}^{2}$ ). With respect to Setup 1, a higher electron energy threshold is imposed to focus on the region where $\Delta \alpha_{\text {had }}(t)$ is larger; 

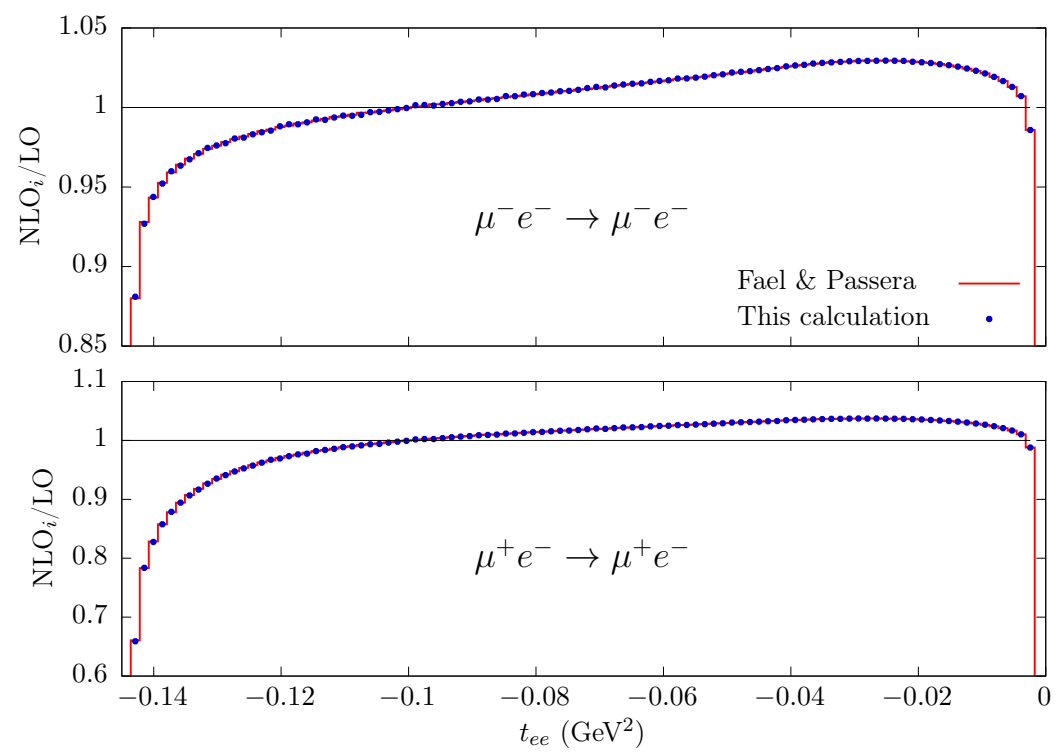

Figure 1. Tuned comparison between our predictions and those of ref. [52] for the ratio of the NLO and LO cross section in QED of the $\mu^{-} e^{-} \rightarrow \mu^{-} e^{-}$(upper panel) and $\mu^{+} e^{-} \rightarrow \mu^{+} e^{-}$(lower panel) process, as a function of the squared momentum transfer $t_{e e}=\left(p_{2}-p_{4}\right)^{2}$.

3. the same criteria as in Setup 1, with an additional acoplanarity cut, applied to partially remove radiative events and thus enhancing the fraction of elastic events. We require acoplanarity $\equiv\left|\pi-\left(\phi_{e}-\phi_{\mu}\right)\right| \leq 3.5 \mathrm{mrad}$ for the sake of illustration;

4. the same criteria as in Setup 2, with the additional acoplanarity cut $\left|\pi-\left(\phi_{e}-\phi_{\mu}\right)\right| \leq$ $3.5 \mathrm{mrad}$.

Notice that $\Delta \alpha_{\text {had }}(t)$ increases as $|t|$ increases and thus, in this kinematical configuration, its largest value corresponds to the small $\theta_{e}$ region. It is also worth noting that all the numerical results are given for so-called bare leptons, i.e. in the absence of lepton-photon recombination criteria, as the details of the measurement of the lepton energies are still under study by the MUonE experiment.

Before entering the discussion of our phenomenological analysis, we show in figure 1 the results of a tuned comparison (i.e. using the same set of input parameters and cuts) between our predictions and those of ref. [52]. The comparison has been performed in order to test the technical accuracy of our calculation and its MC implementation. The results shown in figure 1 correspond to the ratio of the NLO QED differential cross section and the $\mathrm{LO}$ one, both for the process $\mu^{-} e^{-} \rightarrow \mu^{-} e^{-}$(upper panel) and $\mu^{+} e^{-} \rightarrow \mu^{+} e^{-}$(lower panel), as a function of the squared momentum transfer $t_{e e}$.

Figure 1 shows a perfect agreement (within the MC integration statistical error) between the results of the two calculations. In figure 2, the same level of agreement is observed for the ratio above as a function of the squared momentum transfer $t_{\mu \mu}=\left(p_{1}-p_{3}\right)^{2}$. In figures $1-2$ we used Setup 2 with the further constraint that $t_{\mu \mu}$ lies in the same range as $t_{e e}$. 

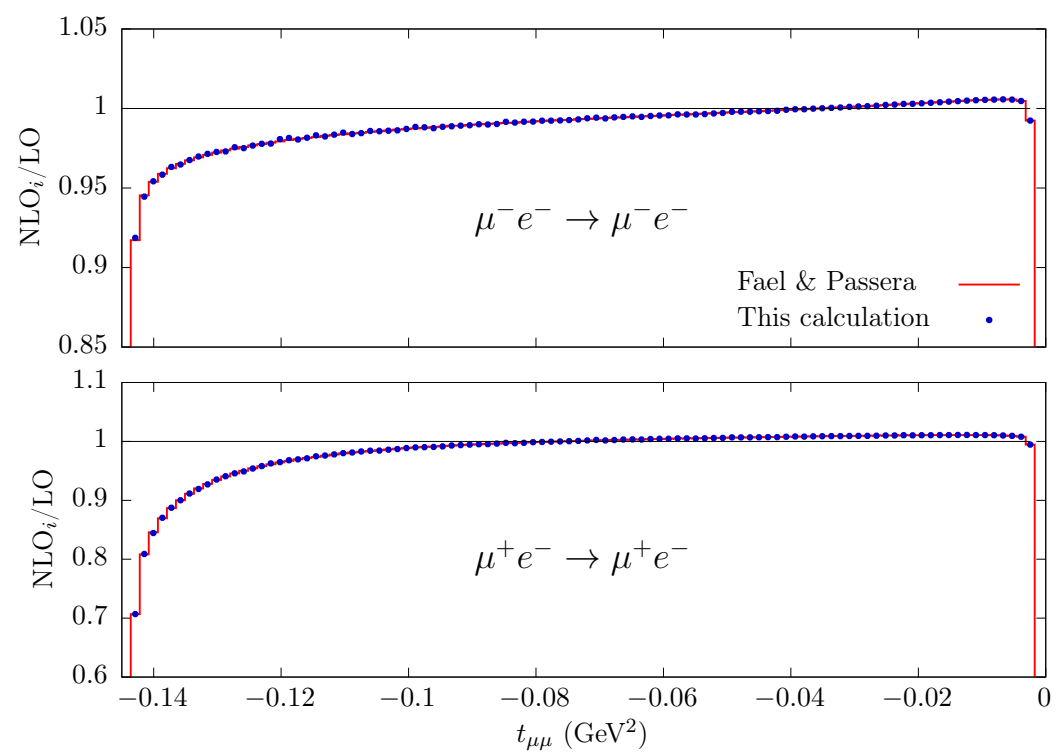

Figure 2. The same as in figure 1, as a function of the squared momentum transfer $t_{\mu \mu}=\left(p_{1}-p_{3}\right)^{2}$.

\subsection{Integrated cross sections}

First, we show numerical results for the radiative corrections at the level of integrated cross section, for the different experimental conditions defined above. We focus on the contributions due to QED corrections and postpone the discussion of the smaller effects due to electroweak contributions to section 3.2.

The cross sections quoted in table 1 are defined as follows:

- $\sigma_{\mathrm{LO}}^{\mathrm{QED}}$ and $\sigma_{\mathrm{NLO}}^{\mathrm{QED}}$ are the QED cross sections at LO and NLO accuracy, ${ }^{7}$ respectively;

- $\sigma_{\mathrm{LO}}^{\mathrm{VP} \text { lep.ttop }}$ is the LO QED cross section including the contribution of the leptonic

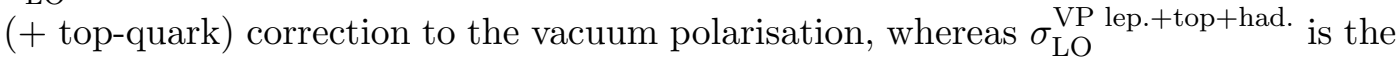
LO QED cross section including the full correction to the running of $\alpha$.

From the above cross section values, we derive the relative corrections shown in table 1 according to the following definitions:

$$
\begin{aligned}
\delta_{\text {lep.+top }}^{\mathrm{VP}} & =\frac{\sigma_{\mathrm{LO}}^{\mathrm{VP} \text { lep.+top }}-\sigma_{\mathrm{LO}}^{\mathrm{QED}}}{\sigma_{\mathrm{LO}}^{\mathrm{QED}}} \quad \delta_{\text {had. }}^{\mathrm{VP}}=\frac{\sigma_{\mathrm{LO}}^{\mathrm{VP}} \text { lep.+top+had. }-\sigma_{\mathrm{LO}}^{\mathrm{VP} \text { lep.+top }}}{\sigma_{\mathrm{LO}}^{\mathrm{VP} \text { lep.top }}} \\
\delta_{\mathrm{NLO}}^{\mathrm{QED}} & =\frac{\sigma_{\mathrm{NLO}}^{\mathrm{QED}}-\sigma_{\mathrm{LO}}^{\mathrm{QED}}}{\sigma_{\mathrm{LO}}^{\mathrm{QED}}} .
\end{aligned}
$$

Generally speaking, the LO cross sections in Setup 1 and Setup 3 differ from those of Setup 2 and Setup 4 because of the different electron energy threshold, which implies a different minimum value of the squared momentum transfer $t$ allowed by the elastic

\footnotetext{
${ }^{7}$ The cross section $\sigma_{\mathrm{NLO}}^{\mathrm{QED}}$ includes the contribution of purely photonic corrections only, as the gaugeinvariant effects of the photon self-energy are evaluated separately in $\sigma_{\mathrm{LO}}^{\mathrm{VP} \text { lep.+top }}$ and $\sigma_{\mathrm{LO}}^{\mathrm{VP} \text { lep.+top+had. }}$
} 


\begin{tabular}{|c|c|c|c|c|}
\hline \multicolumn{5}{|c|}{$\boldsymbol{\mu}^{ \pm} e^{-} \rightarrow \boldsymbol{\mu}^{ \pm} e^{-}$} \\
\hline \multicolumn{3}{|c|}{ Cross section } & Setup $1 \&$ Setup 3 & Setup $2 \&$ Setup 4 \\
\hline \multicolumn{3}{|c|}{$\sigma_{\mathrm{LO}}^{\mathrm{QED}}$} & $1265.060312(7)$ & $245.038910(1)$ \\
\hline \multicolumn{3}{|c|}{$\sigma_{\mathrm{LO}}^{\mathrm{VP} \text { lep.+top }}$} & $1276.876586(9)$ & $247.946485(1)$ \\
\hline \multicolumn{3}{|c|}{$\sigma_{\mathrm{LO}}^{\text {VP lep.+top+had. }}$} & $1276.903841(10)$ & $247.965989(2)$ \\
\hline \multicolumn{5}{|c|}{$\boldsymbol{\mu}^{+} e^{-} \rightarrow \boldsymbol{\mu}^{+} e^{-}$} \\
\hline Cross section & Setup 1 & Setup 2 & Setup 3 & Setup 4 \\
\hline$\sigma_{\mathrm{NLO}}^{\mathrm{QED}}$ & $1325.217(3)$ & $255.8437(5)$ & $1162.447(2)$ & $222.7714(3)$ \\
\hline \multicolumn{5}{|c|}{$\boldsymbol{\mu}^{-} e^{-} \rightarrow \boldsymbol{\mu}^{-} e^{-}$} \\
\hline$\sigma_{\mathrm{NLO}}^{\mathrm{QED}}$ & $1323.478(3)$ & $255.1176(5)$ & $1161.888(1)$ & $222.8545(3)$ \\
\hline \multicolumn{5}{|c|}{$\boldsymbol{\mu}^{ \pm} e^{-} \rightarrow \boldsymbol{\mu}^{ \pm} e^{-}$} \\
\hline \multicolumn{3}{|c|}{ Relative correction } & Setup $1 \&$ Setup 3 & Setup $2 \&$ Setup 4 \\
\hline \multicolumn{3}{|c|}{$\delta_{\text {lep.+top }}^{\mathrm{VP}}$} & $0.009340014(8)$ & $0.011865215(7)$ \\
\hline \multicolumn{3}{|c|}{$\delta_{\text {had. }}^{\mathrm{VP}}$} & $0.000021345(10)$ & $0.000078665(9)$ \\
\hline \multicolumn{5}{|c|}{$\boldsymbol{\mu}^{+} e^{-} \rightarrow \boldsymbol{\mu}^{+} e^{-}$} \\
\hline Relative correction & Setup 1 & Setup 2 & Setup 3 & Setup 4 \\
\hline$\delta_{\mathrm{NLO}}^{\mathrm{QED}}$ & $0.047552(2)$ & $0.044094(2)$ & $-0.081114(1)$ & $-0.090874(1)$ \\
\hline \multicolumn{5}{|c|}{$\mu^{-} e^{-} \rightarrow \mu^{-} e^{-}$} \\
\hline$\delta_{\mathrm{NLO}}^{\mathrm{QED}}$ & $0.046177(2)$ & $0.041130(2)$ & $-0.081556(1)$ & $-0.090535(1)$ \\
\hline
\end{tabular}

Table 1. Cross sections (in $\mu \mathrm{b}$ ) and relative corrections for the processes $\mu^{+} e^{-} \rightarrow \mu^{+} e^{-}$and $\mu^{-} e^{-} \rightarrow \mu^{-} e^{-}$, in the four different setup described in the text. The digits in parenthesis correspond to the $1 \sigma \mathrm{MC}$ error estimate.

kinematics, as remarked above. Going from $E_{e}^{\min }=0.2 \mathrm{GeV}$ to $E_{e}^{\min }=1 \mathrm{GeV}$, the elastic cross section is reduced by about a factor of five.

More in detail, it can be seen from table 1 that the leptonic (+ top) correction to the vacuum polarisation, which is dominated by the electron loop contribution, is of the order of $1 \%$ in all the setup. The hadronic correction to the running of the QED coupling amounts to $2 \times 10^{-5}$ in Setup 1 and Setup 3 and $8 \times 10^{-5}$ in Setup 2 and Setup 4. The impact of $\Delta \alpha_{\text {had }}(t)$ on the differential cross sections, which are the relevant observables for the determination of $a_{\mu}^{\mathrm{HLO}}$ in the MUonE experiment, is shown in section 3.2.

The photonic corrections are positive and rather stable, at the level of $4-5 \%$, when no acoplanarity cuts are applied. They change only by a few per mille when varying the electron energy threshold and are therefore mildly dependent on the applied electron energy cut. On the other hand, they change sign and are enhanced to $8-9 \%$ in the presence of an acoplanarity cut, as a consequence of the increasing importance of soft photon emission. 


\begin{tabular}{|c|c|c|c|c|}
\hline Relative correction & Setup 1 & Setup 2 & Setup 3 & Setup 4 \\
\hline \multicolumn{5}{|c|}{$\boldsymbol{\mu}^{+} \boldsymbol{e}^{-} \rightarrow \boldsymbol{\mu}^{+} \boldsymbol{e}^{-}$} \\
\hline$\delta_{\mathrm{NLO}}^{\text {QED }}$ & $0.04755(1)$ & $0.04409(1)$ & $-0.08111(4)$ & $-0.09087(1)$ \\
\hline$\delta_{\mathrm{NLO}}^{\text {electron }}$ & $0.04694(2)$ & $0.04289(1)$ & $-0.08058(3)$ & $-0.08816(1)$ \\
\hline$\delta_{\mathrm{NLO}}^{\text {muon }}$ & $-0.00008(1)$ & $-0.00028(1)$ & $-0.00078(1)$ & $-0.00256(1)$ \\
\hline$\delta_{\mathrm{NLO}}^{\text {up-down int. }}$ & $0.00069(2)$ & $0.00149(2)$ & $0.00025(5)$ & $-0.00015(2)$ \\
\hline \multicolumn{5}{|c|}{$\boldsymbol{\mu}^{-} \boldsymbol{e}^{-} \rightarrow \boldsymbol{\mu}^{-} \boldsymbol{e}^{-}$} \\
\hline \multicolumn{5}{|c|}{ (QED } \\
\hline$\delta_{\text {NLO }}^{\text {electron }}$ & $0.04618(1)$ & $0.04114(1)$ & $-0.08154(3)$ & $-0.09055(1)$ \\
\hline$\delta_{\mathrm{NLO}}^{\text {muon }}$ & $0.04694(2)$ & $0.04289(1)$ & $-0.08056(2)$ & $-0.08817(1)$ \\
\hline$\delta_{\mathrm{NLO}}^{\text {up-down int. }}$ & $-0.00008(1)$ & $-0.00028(1)$ & $-0.00078(1)$ & $-0.00256(1)$ \\
\hline
\end{tabular}

Table 2. Relative corrections for the gauge-invariant subsets of QED corrections to the processes $\mu^{+} e^{-} \rightarrow \mu^{+} e^{-}$and $\mu^{-} e^{-} \rightarrow \mu^{-} e^{-}$, in the four different setup described in the text. The digits in parenthesis correspond to the $1 \sigma \mathrm{MC}$ error estimate.

Note also that the photonic corrections differ by a few per mille (Setup 1 and Setup 2) and at the $10^{-4}$ level (Setup 3 and Setup 4) for the processes involving opposite charge muons. This aspect is discussed in more detailed in the following.

Within the full set of NLO QED corrections, there are three gauge-invariant subsets of photonic contributions corresponding to:

- virtual and real corrections along the electron line;

- virtual and real corrections along the muon line;

- up-down interference connecting the electron and muon line, due to box contributions and up-down interference of real photon radiation.

The relative contributions associated to the above classes of correction are shown in table 2, where it can be noticed that the corrections due to electron and muon radiation do not change when considering the processes initiated by opposite charge muons. On the other hand, the up-down interference corrections have the same size but opposite sign, as can be expected from the different charge pattern occurring in the interference/box contribution to the two processes. This explains the difference between the NLO QED corrections to the processes with positive and negative muons.

From table 2, one can also see that for both the $\mu^{+} e^{-} \rightarrow \mu^{+} e^{-}$and $\mu^{-} e^{-} \rightarrow \mu^{-} e^{-}$ process the overall (some per cent) corrections are largely dominated by the contribution due to electron radiation. In all the setup, the latter provides more than $95 \%$ of the whole correction. The contributions due to muon radiation and electron-muon interference share the remaining part of the full correction and lie in the range between $10^{-4}$ and $10^{-3}$. In particular, for Setup 1 and Setup 2, the next-to-dominant contribution is given by the up-down interference corrections, whereas in Setup 3 and Setup 4 muon radiation exceeds 


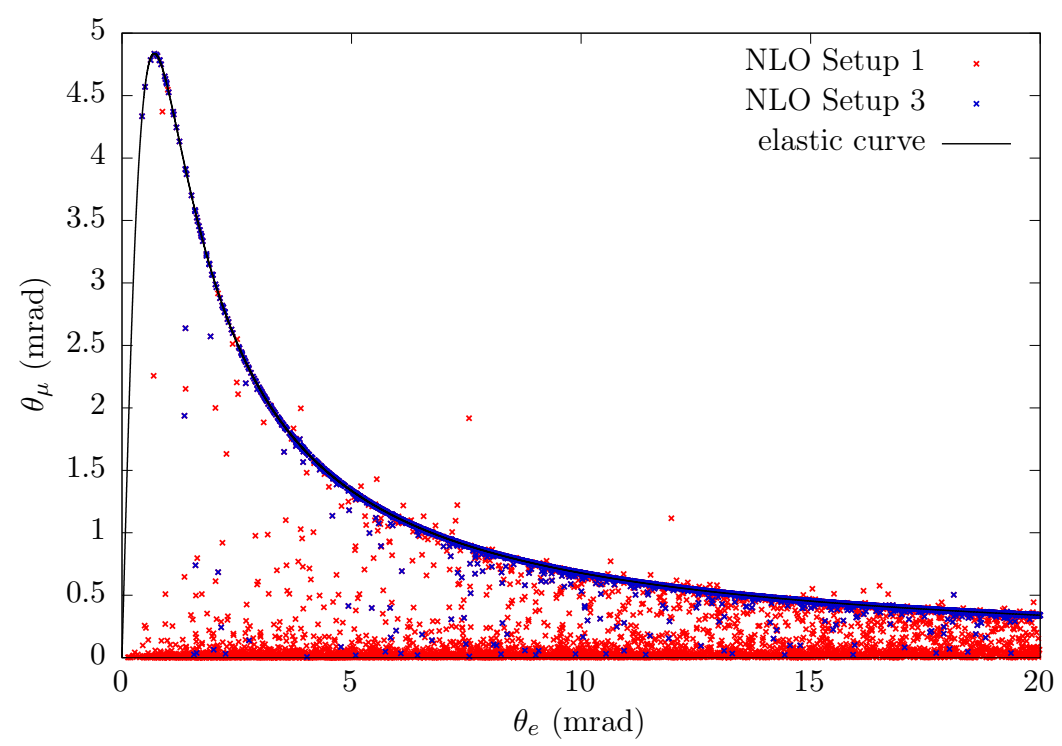

Figure 3. The correlation between the electron scattering angle $\theta_{e}$ and muon scattering angle $\theta_{\mu}$ for the $\mu^{+} e^{-} \rightarrow \mu^{+} e^{-}$process at LO (elastic curve) and NLO QED, for the selection criteria 1 and 3 defined in the text.

the effect of the NLO up-down interference. A more detailed analysis of the contributions due to the different classes of corrections for the differential distributions is given in the next section.

\subsection{Differential cross sections}

More than the integrated cross sections, various distributions are relevant for the newly proposed MUonE experiment. We mainly focus on the $\mu^{+} e^{-} \rightarrow \mu^{+} e^{-}$process because of the larger fraction of positive muons provided by the CERN M2 beam. Particular attention is paid to the differential cross sections as functions of the angular variables, as the proposed experimental arrangement of the MUonE experiment is primarily designed to measure the angles precisely [17].

In figure 3 we compare the correlation, obtained with a sample of MC events, between the scattering angles of the outgoing electron and muon at LO and NLO, for the Setup 1 and Setup 3 defined above. It can be noticed that, in the absence of an acoplanarity cut (Setup 1), the correlation present at LO (elastic curve) is largely modified by the presence of events at relatively small muon angles, which originate from the bremsstrahlung process $\mu^{+} e^{-} \rightarrow \mu^{+} e^{-}+\gamma$. However, the tight acoplanarity cut (Setup 3) turns out to be effective in getting rid of most of these radiative events, thus isolating the elastic correlation curve. Other and more sophisticated elasticity conditions [53] could be applied on the experimental side and easily taken into account at the simulation level.

In figures 4-6 we show the impact of the NLO QED (purely photonic) corrections on a number of differential cross sections of the process $\mu^{+} e^{-} \rightarrow \mu^{+} e^{-}$process, in order to study how the different observables are affected by the radiative corrections and applied cuts. 

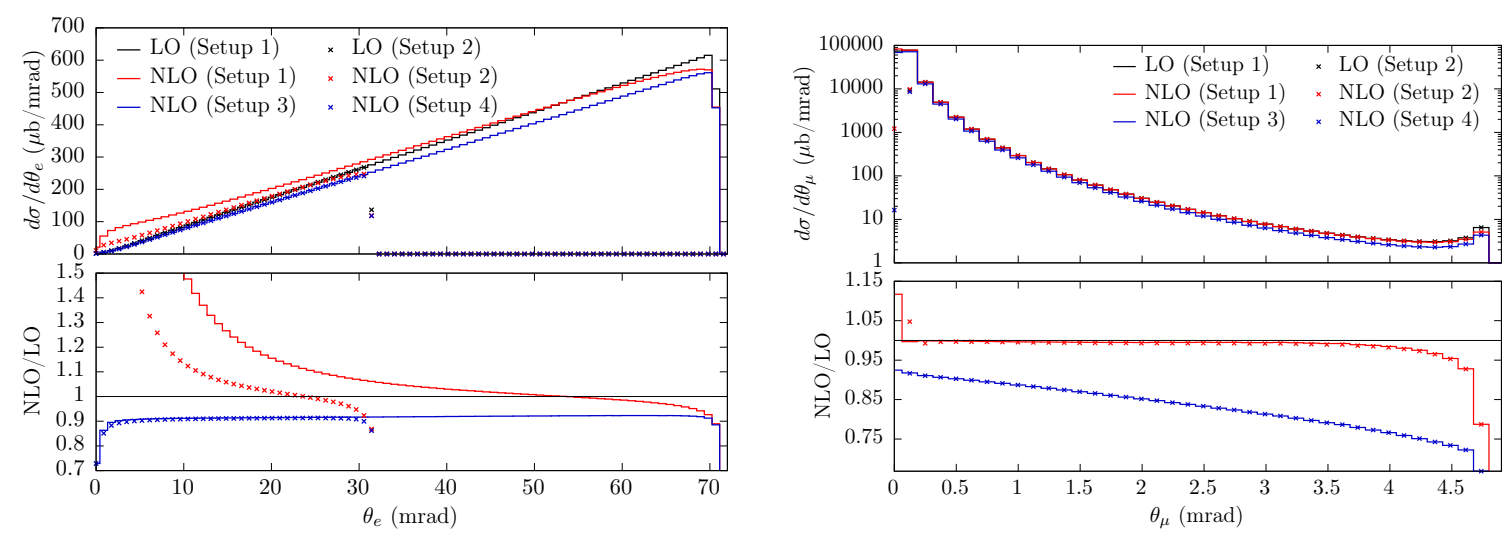

Figure 4. Left plot: the LO and NLO QED cross sections of the $\mu^{+} e^{-} \rightarrow \mu^{+} e^{-}$process, as a function of the electron scattering angle, for the different setup described in the text (upper panel); relative NLO QED corrections (lower panel). Right plot: the same as in the left plot, as a function of the muon scattering angle.

In figure 4 the effect of the QED corrections under the different event selection criteria is shown for the distribution of the electron (left plot) and the muon scattering angles (right plot). It can be clearly seen that, when no elasticity cuts are applied, the electron angle distribution is significantly affected by the QED contributions in the region of small scattering angles, which is the part of the cross section of main interest for the extraction of $\Delta \alpha_{\text {had }}(t)$. This strongly varying distortion, which corresponds to an enhancement of events at relatively small muon angles, can be understood as an effect induced on the electron by the radiative processes $\mu^{+} e^{-} \rightarrow \mu^{+} e^{-}+\gamma$, as already remarked for figure 3 . However, this effect largely disappears if an acoplanarity cut is imposed, thus giving rise to a flat and less significant correction of the order of $10 \%$. It is worth noting that the same does not occur for the $\theta_{\mu}$ distribution. In the absence of an acoplanarity cut, the latter distribution receives a flat and moderate contribution from the radiative corrections (independently of the electron energy threshold), with the exception of the upper hedge where the QED contributions can reach the $20 \%$ level. In the presence of an elasticity condition, the corrections to the muon scattering angle distribution become enhanced and more varying, as a consequence of the more pronounced importance of soft photon radiation.

In figure 5 we show the contribution of the photonic corrections to the differential cross sections as functions of the squared momentum transfer $t_{\mu \mu}$ (left plot) and $t_{e e}$ (right plot). We can notice that, for the situation of acceptance cuts only (Setup 1 and Setup 2), there are small and constant corrections for values $\left|t_{\mu \mu, e e}\right| \lesssim 0.1 \mathrm{GeV}^{2}$. Again, in the presence of an acoplanarity cut, the corrections become larger because of the growing importance of soft photon emission and vary in the range between $10 \%$ and $40 \%$.

Concerning the energy of the outgoing leptons, we show in figure 6 the energy spectrum of the electron (left plot) and of the muon (right plot), respectively. Generally speaking, it can be noticed that the corrections are to a large extent independent of the applied electron energy cut, as they are practically the same for Setup 1 and Setup 2. For these selection criteria, the corrections are slowly varying in almost all the range but they grow 

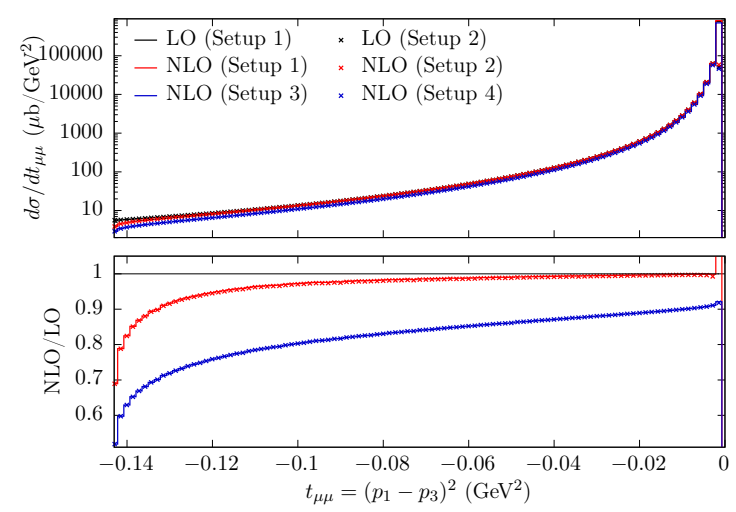
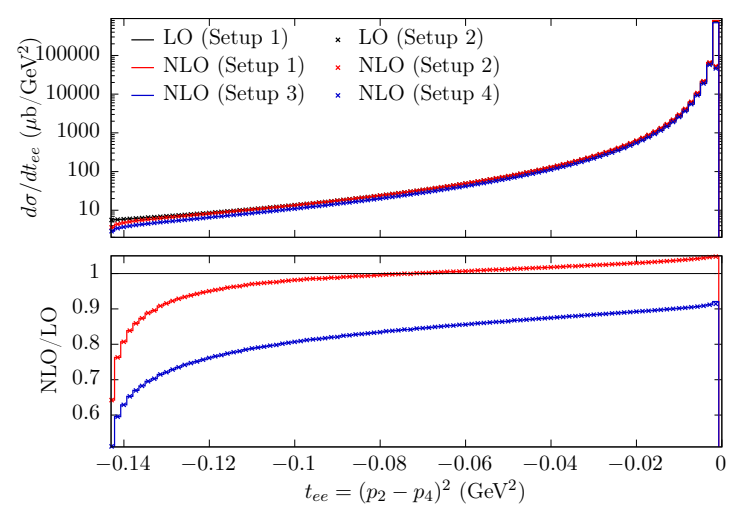

Figure 5. The same as figure 4 for the cross sections as a function of the squared momentum transfer $t_{\mu \mu}$ (left plot) and $t_{e e}$ (right plot).

up to some tens of per cent in the limit of high electron energy and small muon energy. This behaviour can be ascribed to the dominant rôle played by the radiation emitted by the electron leg, which turns out to be emphasised in the above regions by the contribution of soft photons. However, it is worth noting that the cross sections corresponding to the above kinematical limits are pretty small. When an elasticity condition is imposed, the QED corrections to the lepton energies lie in the range between $10 \%$ and $50 \%$.

We come now to the discussion of the effects due to the electroweak contributions and vacuum polarisation. We also examine in detail the impact of the gauge-invariant subsets of photonic corrections at the level of distributions, thus completing the analysis presented in section 3.1 for the integrated cross sections. We provide results which are valid for the processes $\mu^{ \pm} e^{-} \rightarrow \mu^{ \pm} e^{-}$and are given in figures $7-11$.

In figure 7 the size of the electroweak contributions at LO and NLO accuracy is shown for the cross sections $d \sigma / d \theta_{e}$ (left plot) and $d \sigma / d \theta_{\mu}$ (right plot) of the processes $\mu^{ \pm} e^{-} \rightarrow$ $\mu^{ \pm} e^{-}$. The same effects are shown in figure 8 for the cross sections as a function of the squared momentum transfer $t_{\mu \mu}$ and $t_{e e}$. In order to consistently disentangle pure electroweak effects, we define $\Delta_{\mathrm{EW}}^{\mathrm{LO}}$ and $\Delta_{\mathrm{EW}}^{\mathrm{NLO}}$ as follows

$$
\Delta_{\mathrm{EW}}^{\mathrm{LO}}=\frac{d \sigma_{\mathrm{EW}}^{\mathrm{LO}}-d \sigma_{\mathrm{QED}}^{\mathrm{LO}}}{d \sigma_{\mathrm{QED}}^{\mathrm{LO}}} \quad \Delta_{\mathrm{EW}}^{\mathrm{NLO}}=\frac{\left(d \sigma_{\mathrm{EW}}^{\mathrm{NLO}}-d \sigma_{\mathrm{EW}}^{\mathrm{LO}}\right)-\left(d \sigma_{\mathrm{QED}}^{\mathrm{NLO}}-d \sigma_{\mathrm{QED}}^{\mathrm{LO}}\right)}{d \sigma_{\mathrm{QED}}^{\mathrm{NLO}}} .
$$

From figures 7-8, it can be noticed that the LO electroweak contributions to the observables of the $\mu^{+} e^{-} \rightarrow \mu^{+} e^{-}$process (upper panels) have a different shape and sign with respect to the same observables of the $\mu^{-} e^{-} \rightarrow \mu^{-} e^{-}$process (lower panels). This asymmetric behaviour is a parity-violation effect induced by the presence of the $Z$-exchange contribution in the LO diagrams. From figure 7 , one can also see that the $\gamma-Z$ tree-level contributions are at the $10^{-5}$ level for small electron angles and relatively large muon angles. Effects of the same order are observed in figure 8 for the cross sections as a function of the momentum transfer $t_{\mu \mu}$ and $t_{e e}$ for $\left|t_{\mu \mu, e e}\right|$ values larger than about $0.1 \mathrm{GeV}^{2}$. In view of the target accuracy of the experiment, we can conclude that the LO electroweak contributions must be taken into account in any calculation aiming at a $10 \mathrm{ppm}$ precision. Concerning 

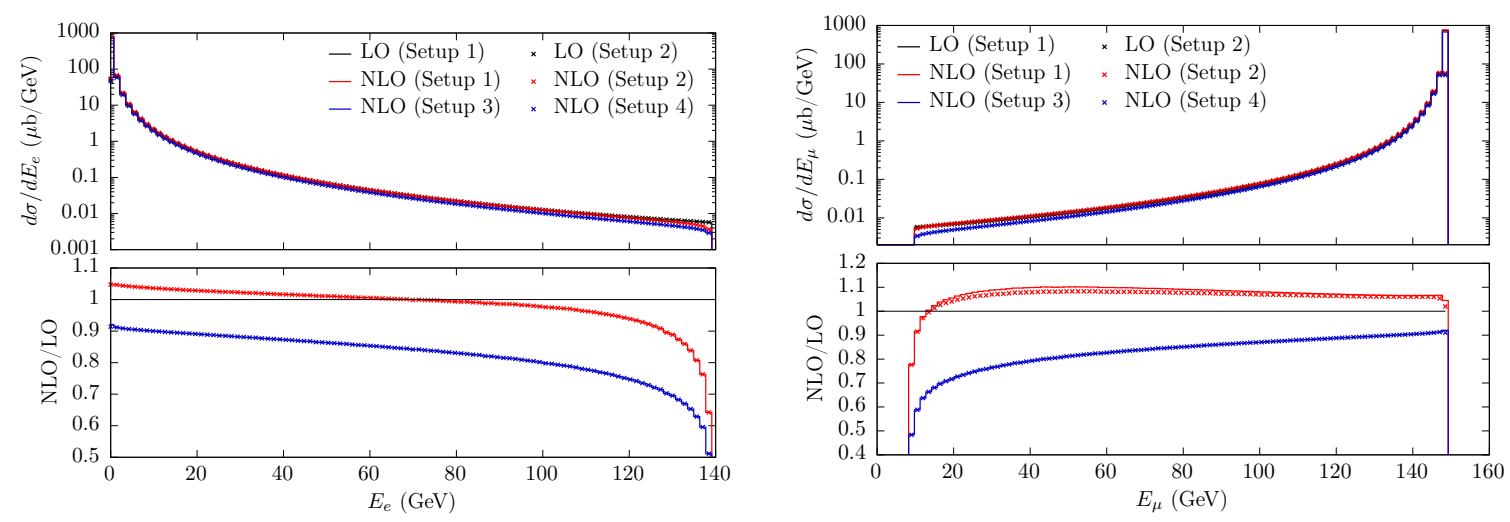

Figure 6. The same as figure 4 for the cross sections as a function of the electron (left plot) and muon (right plot) energy.
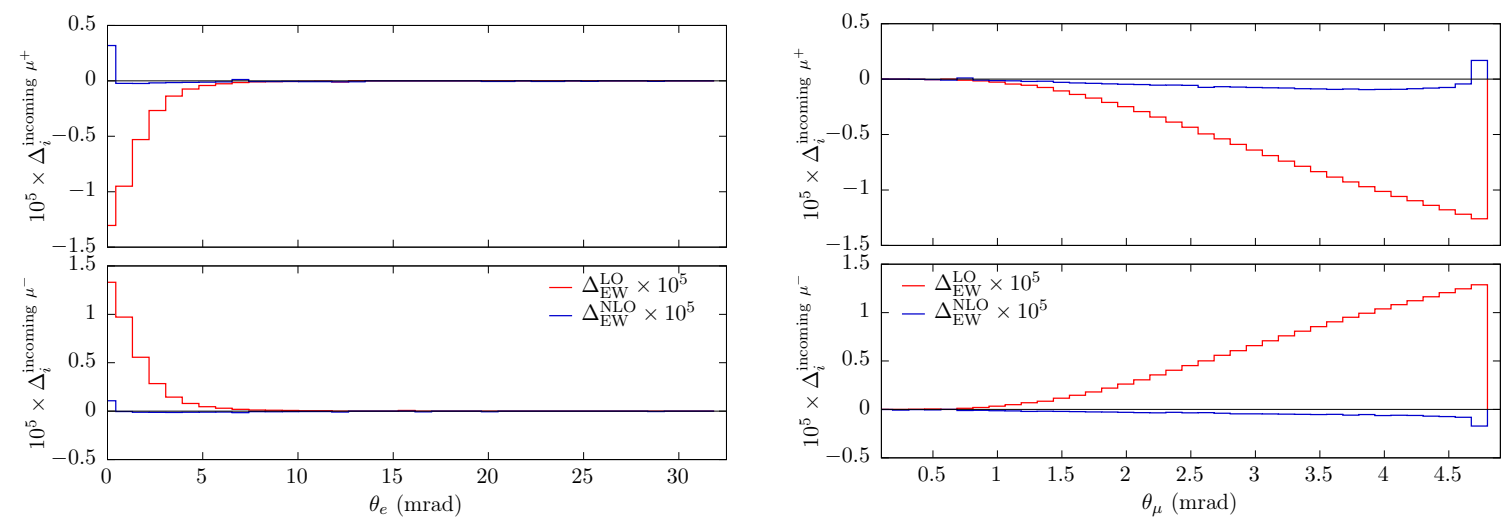

Figure 7. Left plot: the LO and NLO electroweak contributions to the cross section of the process $\mu^{+} e^{-} \rightarrow \mu^{+} e^{-}$(upper panel) and of the process $\mu^{-} e^{-} \rightarrow \mu^{-} e^{-}$(lower panel), as a function of the electron scattering angle. Right plot: the same as in the left plot as a function of the muon scattering angle. The results refer to Setup 2 described in the text.

the impact of the NLO electroweak corrections, it can be seen that they are almost flat and negligible for all the differential cross sections, their contribution being well below $10^{-5}$. We checked that also for the electron and muon energy the LO electroweak contributions can reach the $10 \mathrm{ppm}$ level, the one-loop electroweak corrections being always negligible.

According to the proposed strategy of the MUonE experiment, the leading hadronic contribution to the muon $g-2$ can be derived after subtracting the leptonic correction to the running of the QED coupling as extracted from the data [17]. Therefore, it is important to establish the accuracy in the calculation of the leptonic contributions to the vacuum polarisation. To this end, we show in figure 9 the behaviour of the leptonic corrections to the running of $\alpha$ at the different perturbative orders [33], as a function of the squared momentum transfer $t$ (left plot) and of the Feynman-parameter variable $x$ (right plot). As a reference, also the hadronic contribution is shown, as calculated using the dispersive approach available in the hadr5n16.f routine [36]. The results as a function of $x$ are shown since the contribution $a_{\mu}^{\mathrm{HLO}}$ can be obtained in the space-like approach as a one- 

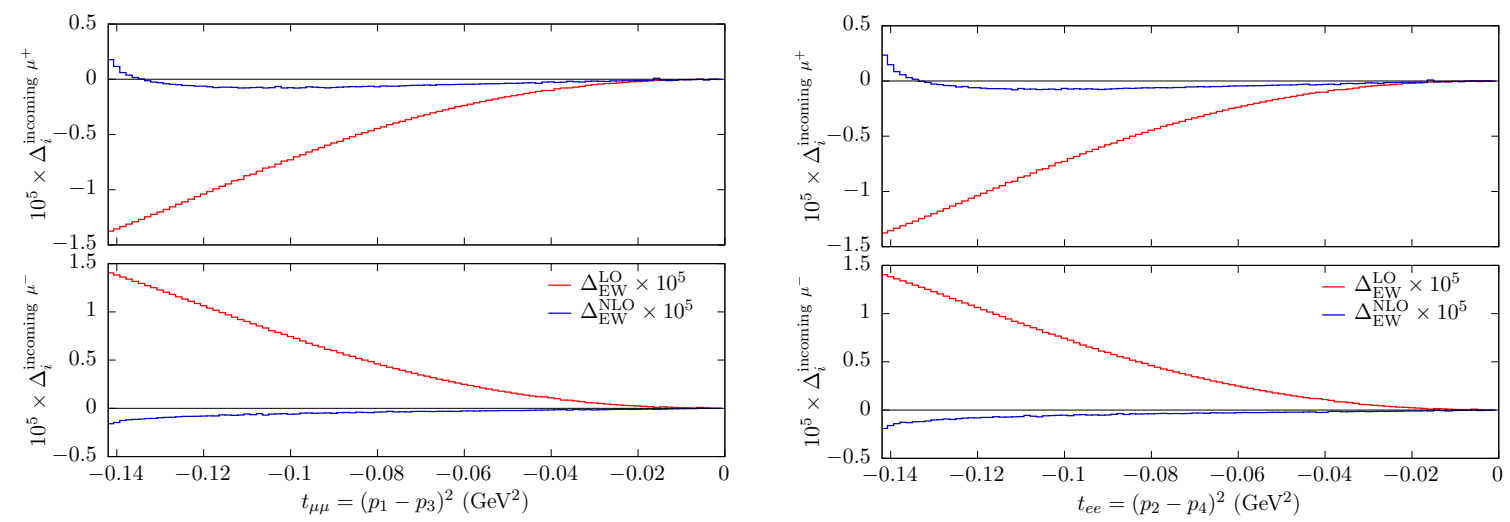

Figure 8. The same as figure 7 for the cross sections of the processes $\mu^{ \pm} e^{-} \rightarrow \mu^{ \pm} e^{-}$, as functions of the squared momentum transfer $t_{\mu \mu}$ (left plot) and $t_{e e}$ (right plot).
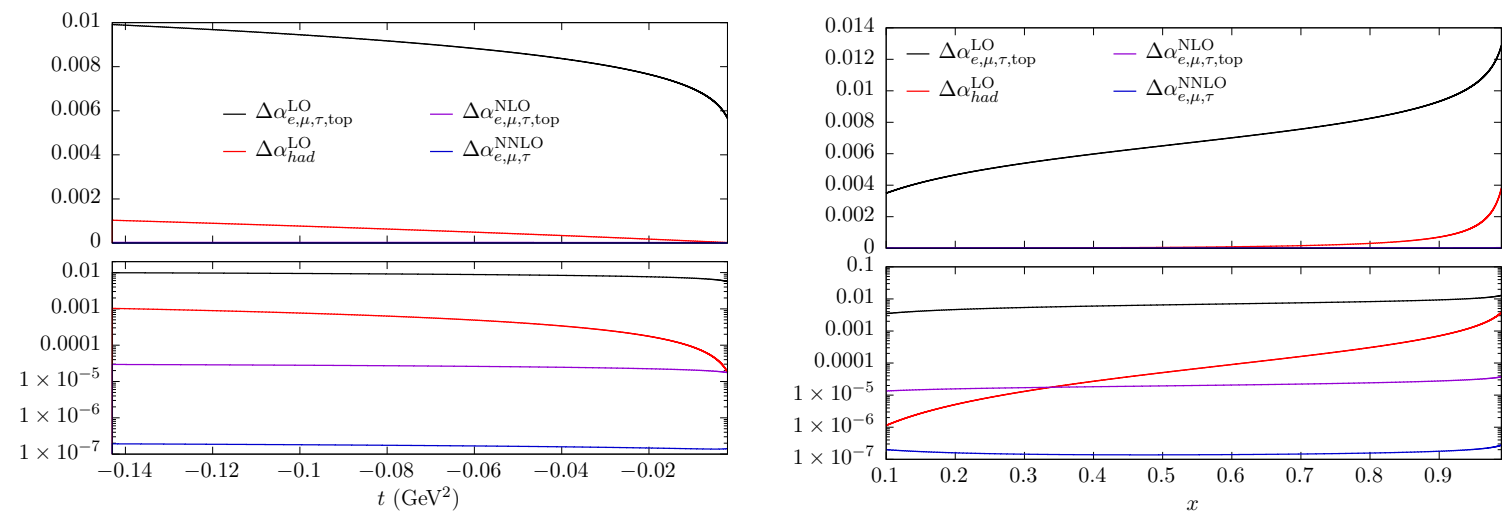

Figure 9. Left plot: the leptonic ( + top) correction and the hadronic correction to the running of $\alpha_{\mathrm{QED}}$, as a function of the squared momentum transfer $t$ in linear (upper panel) and log scale (lower panel). Right plot: the same as in the left plot as a function of the Feynman variable $x$.
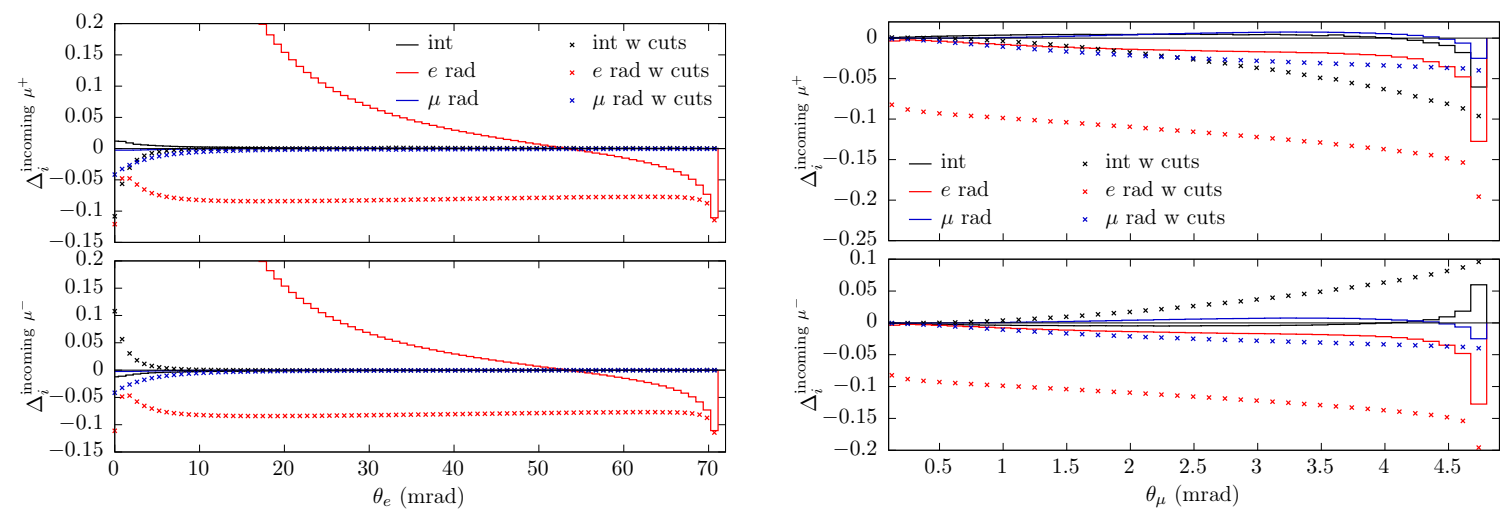

Figure 10. Left plot: the contribution of the QED gauge-invariant subsets of photonic corrections to the cross section of the process $\mu^{+} e^{-} \rightarrow \mu^{+} e^{-}$(upper panel) and of the process $\mu^{-} e^{-} \rightarrow \mu^{-} e^{-}$ (lower panel), as a function of the electron scattering angle. Right plot: the same as in the left plot as a function of the muon scattering angle. The results refer to Setup 1 and Setup 3 described in the text. 

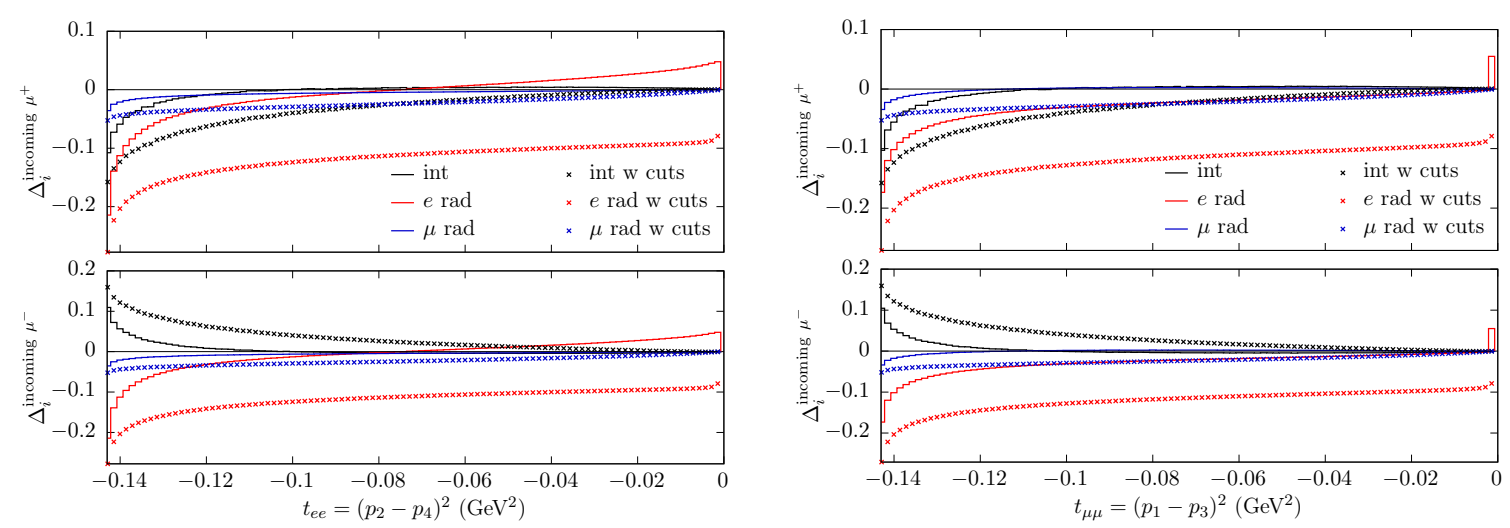

Figure 11. The same as figure 10 for the cross sections for the cross sections of the processes $\mu^{ \pm} e^{-} \rightarrow \mu^{ \pm} e^{-}$, as a function of the squared momentum transfer $t_{\mu \mu}$ (left plot) and $t_{e e}$ (right plot).

dimensional integral over the variable $x$ involving the hadronic correction to $\alpha_{\mathrm{QED}}$ evaluated at $t(x)=x^{2} m_{\mu}^{2} /(x-1)[15,17]$. As can be seen, the leptonic corrections at LO (one-loop approximation) vary from $1 \%$ at large $|t|$ and $x$ values to some per mille at small $|t|$ and $x$. The NLO (two-loop accuracy) and NNLO (three-loop approximation) leptonic corrections are of the order of $10^{-5}$ and $10^{-7}$, respectively. Therefore, the present knowledge of the above corrections does not provide a limitation to the proposed experimental strategy. The hadronic contribution varies from $0.1 \%$ at relatively large $t$ and $x$ values, which define the "signal region" of the experiment, to the $10^{-5}-10^{-6}$ level at small $t$ and $x$, where the cross section of the process can be used as normalisation.

An examination of the different sources of photonic corrections is shown for the $d \sigma / d \theta_{e}$ and for $d \sigma / d \theta_{\mu}$ in figure 10 and the $d \sigma / d t_{\mu \mu}$ and $d \sigma / d t_{e e}$ in figure 11. The upper(lower) panels refer to the $\mu^{+} e^{-} \rightarrow \mu^{+} e^{-}\left(\mu^{-} e^{-} \rightarrow \mu^{-} e^{-}\right)$process. This analysis is performed in order to understand how the different gauge-invariant classes contribute to the overall QED corrections previously discussed. The main message that can be drawn by inspection of figure 10 and figure 11 is that the overall QED correction over the full range is, in general, the result of a delicate interplay between the various sources of radiation. A further important and general remark is that the corrections due to up-down interference are of opposite sign for the two processes, which is the origin of the different overall correction for the processes involving positive and negative muons. Moreover, one can notice that the interference contribution plays a significant rôle in the region of small electron scattering angles, i.e. for large $\left|t_{\mu \mu, e e}\right|$ values. This behaviour has to be ascribed to the presence in the up-down interference of logarithmic (and squared logarithmic) angular contributions of the type $\ln (u / t)$, which become potentially enhanced when either $t$ or $u$ are small.

More in detail, one can see from figure 10 that, in the presence of acceptance cuts only, the correction to the electron angle distribution is completely dominated by the contribution of electron radiation, the other effects being almost flat and much smaller over the full range. However, if an acoplanarity cut is applied, the contributions due to muon radiation and up-down interference corrections become visible in the region of small electron scattering angles, where they amount to some per cent. Interestingly, the above 
contributions have the same sign in the $\mu^{+} e^{-} \rightarrow \mu^{+} e^{-}$process (upper panel of the left plot) and sum up to contribute to the overall QED correction, whereas they tend to cancel in the $\mu^{-} e^{-} \rightarrow \mu^{-} e^{-}$process (lower panel of the left plot). As far as the $d \sigma / d \theta_{\mu}$ cross section is concerned, it can be noticed that, both in the absence and in the presence of acoplanarity cuts, all the contributions play a rôle, especially in the region of relatively large muon angles around $4.5 \mathrm{mrad}$. In this region, the different sets of correction vary between $5 \%$ and $20 \%$, the dominant contribution being electron radiation, followed by the interference correction and muon radiation, respectively. Like for the $d \sigma / d \theta_{e}$ cross section, all the corrections have the same sign in the final part of the spectrum of the $\mu^{+} e^{-} \rightarrow \mu^{+} e^{-}$ process (upper panel of the right plot), whereas for the $\mu^{-} e^{-} \rightarrow \mu^{-} e^{-}$process (lower panel of the right plot) the positive up-down interference contributions tend to compensate the negative effect of the electron and muon radiation. Similar considerations apply to the $d \sigma / d t_{\mu \mu}$ and $d \sigma / d t_{e e}$ distributions shown in figure 11. All in all, these results indicate that all the sources of corrections have to be taken into account for precise predictions for the differential cross sections.

We now discuss finite electron mass corrections. We are interested in assessing the (IR-safe) effects due to taking the limit of vanishing electron mass in the virtual (and real soft-photon) amplitudes. In order to achieve the aim, we adopt the following procedure to approximate the ingredients of the complete NLO QED correction:

- we keep fully massive momenta. The choice is driven by the fact that with a massless electron its rest frame (i.e. the lab frame) could not be defined. We therefore keep fully massive phase-space and flux factor. We then choose to express the $2 \rightarrow 2$ amplitudes in terms of the independent $s$ and $t$ Mandelstam invariants;

- for virtual corrections, we completely reduce the tensor structures to scalar functions. We then retain exact $m_{e}$ dependency in those terms enhanced by logarithms of the artificial IR parameter $\lambda$. In the non-IR remainder, we express $u=2 m_{\mu}^{2}-s-t$ (i.e. discarding $2 m_{e}^{2}$ ) and we neglect any $m_{e}^{2}$ contribution except in the arguments of the collinear logarithms;

- for real soft-photon corrections, we proceed similarly: we keep exact $m_{e}$ dependency in terms proportional to $\log \left(\omega_{s} / \lambda\right)$ and neglect any $m_{e}$ corrections in the remainder, except in the arguments of the collinear logarithms;

- for real corrections, we do not make any approximation, because in this case finite mass corrections emerge from a delicate interplay between matrix elements and integration over the 3 -body phase space.

We remark that with these choices our $m_{e} \rightarrow 0$ limit is always independent of the IR parameters $\lambda$ and $\omega_{s}$ by construction and gives an estimate of the effects due to neglecting $m_{e}$ in the one-loop virtual amplitudes. We also stress that also in our massless limit terms proportional to $\log ^{2}\left(Q^{2} / m_{e}^{2}\right)$ are cancelled out between virtual and real soft-photon corrections, as in the exact calculation. 

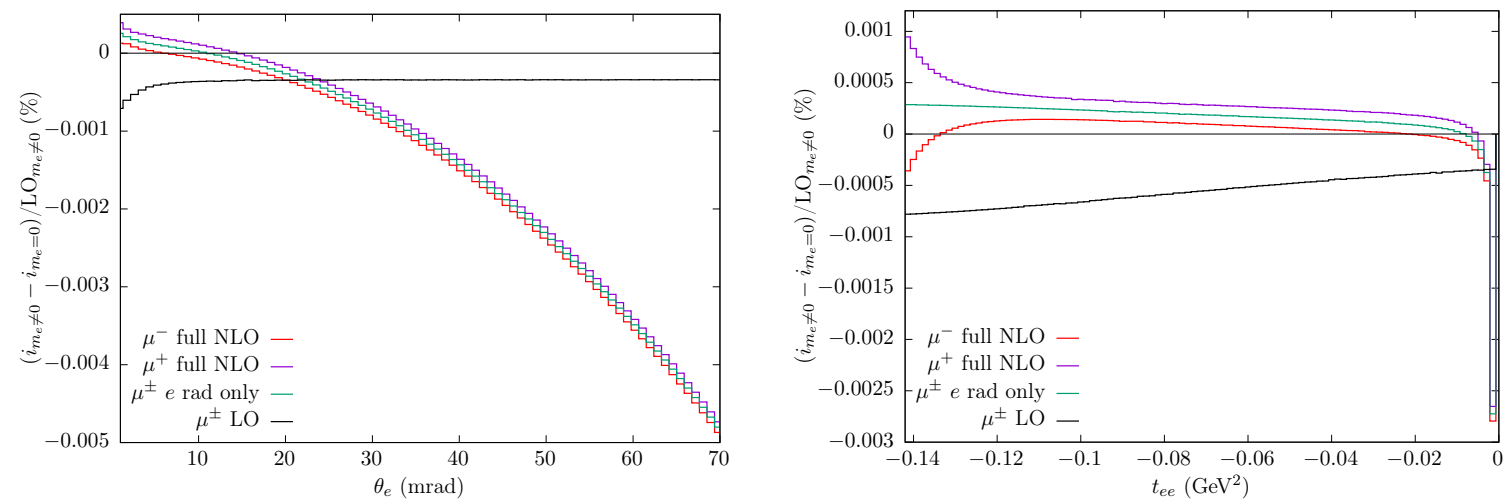

Figure 12. Left plot: the contribution of the electron mass corrections to the cross section of the processes $\mu^{ \pm} e^{-} \rightarrow \mu^{ \pm} e^{-}$, as a function of the electron scattering angle. Right plot: the same as in the left plot as a function of the squared momentum transfer $t_{e e}$. The predictions refer to Setup 1 defined in the text.

The method outlined above has been applied in the computation of the full NLO QED corrections and of the gauge-invariant subset given by the electron-line (virtual and real) contributions. The comparison between the exact results of section 2.1 and those obtained in the $m_{e} \rightarrow 0$ limit provides an IR-safe estimate of finite electron mass effects.

In figure 12 we show our results for Setup 1, in per cent of the fully massive LO differential cross section. The predictions refer to both incoming $\mu^{+}$and $\mu^{-}$and are just shown for the electron scattering angle and the squared momentum transfer $t_{e e}$, for the sake of illustration. Similar results apply to the other distributions considered in our study.

From figure 12, it can be noticed that the contribution of $m_{e}$-dependent terms to the LO cross section is almost flat and below the $10 \mathrm{ppm}$ level. In the considered Setup, the electron-mass corrections at NLO contribute to $d \sigma / d \theta_{e}$ and $d \sigma / d t_{e e}$ in the range from a few to some $10^{-5}$, getting larger as $\theta_{e}$ increases. We notice that the largest part of the finite $m_{e}$ corrections is due to radiation from the electron line only, the full correction lying around it. The extra corrections with respect to the electron line only are dominated by up-down interference and box diagrams.

To conclude, we show in figure 13 and in figure 14 numerical results for the ratios defined as follows:

$$
R_{i}=\frac{d \sigma_{i}\left(\Delta \alpha_{\text {had. }}(t) \neq 0\right)}{d \sigma_{i}\left(\Delta \alpha_{\text {had. }}(t)=0\right)} .
$$

According to eq. (3.3), $R_{i}$ is defined as the ratio between a generic cross section including the contribution of the hadronic correction to the running of $\alpha$ and the same cross section without it. As such, this quantity gives the sensitivity of a given observable to the signal of interest for the MUonE experiment. We study how the ratios $R_{i}$ are affected by the QED corrections under the different selection criteria and for the two processes $\mu^{ \pm} e^{-} \rightarrow \mu^{ \pm} e^{-}$.

The results of this study are shown in figure 13 as a function of the electron (left plot) and muon (right plot) scattering angles. Figure 14 illustrates the situation as a function of the squared momentum transfer $t_{\mu \mu}$ (left plot) and $t_{e e}$ (right plot). As can be seen, there is 

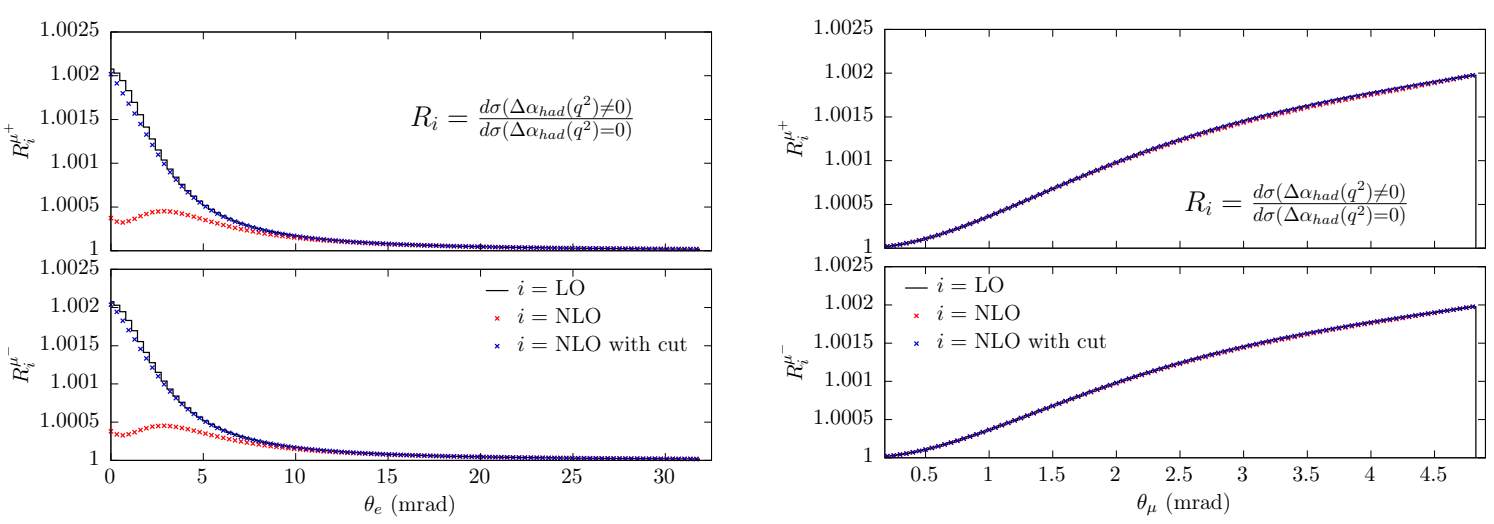

Figure 13. Left plot: the ratios $R_{i}$ defined in the text for the process $\mu^{+} e^{-} \rightarrow \mu^{+} e^{-}$(upper panel) and the process $\mu^{-} e^{-} \rightarrow \mu^{-} e^{-}$(lower panel), as a function of the electron scattering angle. Right plot: the same as in the left plot as a function of the muon scattering angle. The results refer to Setup 2 and Setup 4 described in the text.
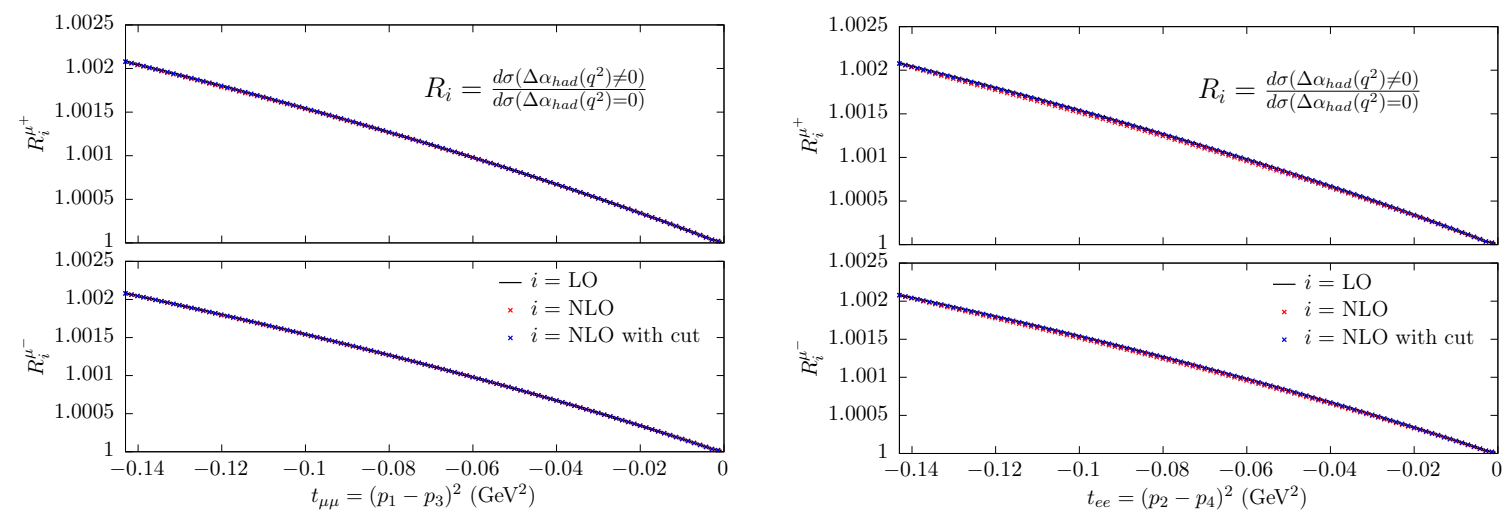

Figure 14. The same as in figure 13 as a function of the squared momentum transfer $t_{\mu \mu}$ (left plot) and $t_{e e}$ (right plot).

not an appreciable difference between the results valid for the process initiated by positive muons (upper panels) and those involving negative muons (upper panels).

From figure 13, it is particularly evident that, in the absence of elasticity conditions (Setup 2), the ratio as a function of the electron scattering angle is strongly affected by the contribution of QED radiation, especially in the region of small angles, where the highest sensitivity, at the per mille level, is present. However, this sensitivity is largely recovered by applying an acoplanarity cut (Setup 4), which removes the contribution of the radiative processes. On the other hand, the ratio as a function of the muon scattering angle appears to be particularly robust under the contribution of the radiative corrections and applied cuts. The same property is shared by the ratios as functions of $t_{\mu \mu}$ and $t_{e e}$, as can be seen from figure 14 .

\section{Conclusions}

In this work, we have computed the full set of NLO corrections to $\mu^{ \pm} e^{-} \rightarrow \mu^{ \pm} e^{-}$scattering in the SM. The study is motivated by the recent proposal of measuring the effective 
electromagnetic coupling constant in the space-like region by using this process (MUonE experiment). From the measurement of the hadronic contribution to the running of $\alpha_{\mathrm{QED}}$, the leading hadronic contribution to the muon anomaly can be derived according to an alternative approach to the standard time-like evaluation of $a_{\mu}^{\mathrm{HLO}}$.

In view of the challenging target accuracy (at the $10 \mathrm{ppm}$ level), we have computed the NLO QED and purely weak corrections without any approximation and developed a corresponding fully differential MC code, which is available for first experimental studies.

We have performed a comprehensive phenomenological analysis of the $\mu e \rightarrow \mu e$ process at NLO accuracy under different event selection conditions, in order to study the dependence of the radiative corrections on the applied cuts. To this end, we have provided detailed results both at the level of integrated cross sections and distributions.

We have shown that the NLO electroweak corrections are largely dominated by the QED effects, the contribution of the NLO purely weak corrections being typically well below $10^{-5}$ and hence negligible. Only the tree-level $\gamma+Z$ contribution plays a rôle, as its contribution can reach the $10^{-5}$ level in particularly important kinematical regions. Therefore, a first conclusion of our study is that at least the LO electroweak contributions are necessary to achieve the required theoretical accuracy.

We have also scrutinised the accuracy in the calculation of the leptonic corrections to the vacuum polarisation, since, according to the proposed strategy of the MUonE experiment, the leading hadronic contribution to the muon anomaly is obtained after subtraction of the leptonic contribution to the running of the QED coupling. We have shown that the leptonic corrections beyond the one-loop approximation, i.e. at two-loop and three-loop accuracy, are of the order of $10^{-5}$ and $10^{-7}$, respectively, and therefore are not a limitation to the proposed experimental procedure.

We have examined the impact of the photonic corrections to all the observables of experimental interest, by considering some variants of possible experimental cuts. We have pointed out that, by assuming the presence of acceptance cuts only, the integrated cross sections receive a moderate correction, of about 5\%. By imposing an elasticity condition given by a tight acoplanarity cut, the QED corrections to the integrated cross sections change sign and rise to the $10 \%$ level, as a consequence of the increased importance of soft photon emission.

We have analysed how the differential cross sections are affected by the QED corrections under the different selection criteria. We have shown that the electron angle distribution receives an extremely important and strongly varying correction, when no elasticity cuts are applied. This behaviour is due to a large kinematical effect induced on the electron scattering angle by the processes accompanied by real photon emission. However, in such an experimental configuration, the corrections to the other distributions are much less pronounced and slowly varying. We have demonstrated that a simple acoplanarity cut is of great help in getting rid of most of the radiative processes, which populate the region of relatively small muon angles, and singles out elastic events. The application of this cut tends to stabilise the QED contribution to the electron angle distribution, giving rise to a flat and less significant correction of the order of $10 \%$. However, as a drawback, the acoplanarity cut enhances the contribution of the photonic corrections to the other differential cross 
sections, because of the growing importance of soft photon radiation. More sophisticated elasticity conditions are being studied at the experimental level and simulated accordingly, in order to optimise the dependence of the radiative corrections on the applied cuts.

We have studied how the different gauge-invariant subsets of photonic corrections contribute to the overall QED contribution. We have shown that, in general, the radiation by the electron leg is the dominant contribution but also emphasised that all the sources of corrections play a rôle for a correct description of the distributions. In particular, we have shown that the corrections due to up-down interference and box contributions are of opposite sign for the processes involving opposite charge muons, thus explaining the difference between the corrections affecting the $\mu^{+} e^{-} \rightarrow \mu^{-} e^{-}$and $\mu^{-} e^{-} \rightarrow \mu^{-} e^{-}$ scattering. In particular, we have pointed out that the interference corrections provide a significant contribution for small electron scattering angles or, equivalently, at relatively large values of the squared momentum transfer, which defines the signal region of the MUonE experiment.

We have also given an estimate of the finite electron-mass corrections, to show that they are not negligible at NLO accuracy and lie in the range from a few to some $10^{-5}$, being dominated by the corrections from the electron leg.

In conclusion, we have also pointed out that, independently of the applied cuts, the cross section as a function of the muon scattering angle is a particularly robust observable under the contribution of the radiative corrections for the extraction of the signal of interest. However, also the electron angle distribution can be used to this end, if appropriate elasticity cuts are applied.

The results here presented represent the first step towards the realisation of a highprecision theoretical tool necessary for the analysis of the data of the MUonE experiment. In the near future, we plan to match the NLO QED corrections to $\mu e \rightarrow \mu e$ with the contribution due to multiple photon emission, following the formulation successfully applied to Bhabha scattering and QED processes at flavour factories [54-56], Drell-Yan processes at hadron colliders [57, 58] and Higgs boson decay into four leptons [59]. Over the longer term, we are interested in developing a MC event generator including NNLO corrections and resummation, which will be ultimately needed for data analysis.

\section{Acknowledgments}

We are sincerely grateful to all our MUonE colleagues for stimulating collaboration and many useful discussions, which are the framework of the present study. We are indebted to Matteo Fael and Massimo Passera for providing us with the results of their calculation used in the tuned comparison shown in the paper.

We thank for its hospitality and its partial support the Mainz Institute for Theoretical Physics (MITP), where part of the present work has been carried out during the topical workshop "The evaluation of the leading hadronic contribution to the muon anomalous magnetic moment" held in February 2018. 
Open Access. This article is distributed under the terms of the Creative Commons Attribution License (CC-BY 4.0), which permits any use, distribution and reproduction in any medium, provided the original author(s) and source are credited.

\section{References}

[1] F. Jegerlehner, The Anomalous Magnetic Moment of the Muon, Springer Tracts Mod. Phys. 274 (2017) 1 [INSPIRE].

[2] F. Jegerlehner and A. Nyffeler, The Muon g-2, Phys. Rept. 477 (2009) 1 [arXiv:0902.3360] [INSPIRE].

[3] Muon G-2 collaboration, Final Report of the Muon E821 Anomalous Magnetic Moment Measurement at BNL, Phys. Rev. D 73 (2006) 072003 [hep-ex/0602035] [INSPIRE].

[4] F. Jegerlehner, The Muon g- 2 in Progress, Acta Phys. Polon. B 49 (2018) 1157 [arXiv: 1804.07409] [INSPIRE].

[5] M. Knecht, General discussion on g-2, EPJ Web Conf. 179 (2018) 01008 [INSPIRE].

[6] A. Keshavarzi, D. Nomura and T. Teubner, Muon $g-2$ and $\alpha\left(M_{Z}^{2}\right)$ : a new data-based analysis, Phys. Rev. D 97 (2018) 114025 [arXiv: 1802.02995] [INSPIRE].

[7] M. Davier, A. Hoecker, B. Malaescu and Z. Zhang, Hadron Contribution to Vacuum Polarisation, Adv. Ser. Direct. High Energy Phys. 26 (2016) 129 [INSPIRE].

[8] Muon G-2 collaboration, Muon $(g-2)$ Technical Design Report, arXiv:1501.06858 [INSPIRE].

[9] J-PARC G-2 collaboration, Measurement of muon $g-2$ and EDM with an ultra-cold muon beam at J-PARC, Nucl. Phys. Proc. Suppl. 218 (2011) 242 [inSPIRE].

[10] M. Della Morte et al., The hadronic vacuum polarization contribution to the muon $g-2$ from lattice QCD, JHEP 10 (2017) 020 [arXiv:1705.01775] [INSPIRE].

[11] H.B. Meyer and H. Wittig, Lattice QCD and the anomalous magnetic moment of the muon, Prog. Part. Nucl. Phys. 104 (2019) 46 [arXiv:1807.09370] [INSPIRE].

[12] Budapest-Marseille-Wuppertal collaboration, Hadronic vacuum polarization contribution to the anomalous magnetic moments of leptons from first principles, Phys. Rev. Lett. 121 (2018) 022002 [arXiv:1711.04980] [INSPIRE].

[13] RBC and UKQCD collaborations, Calculation of the hadronic vacuum polarization contribution to the muon anomalous magnetic moment, Phys. Rev. Lett. 121 (2018) 022003 [arXiv: 1801.07224] [INSPIRE].

[14] D. Giusti, F. Sanfilippo and S. Simula, Light-quark contribution to the leading hadronic vacuum polarization term of the muon $g-2$ from twisted-mass fermions, Phys. Rev. D 98 (2018) 114504 [arXiv:1808.00887] [INSPIRE].

[15] C.M. Carloni Calame, M. Passera, L. Trentadue and G. Venanzoni, A new approach to evaluate the leading hadronic corrections to the muon g-2, Phys. Lett. B 746 (2015) 325 [arXiv: 1504.02228] [INSPIRE].

[16] B.E. Lautrup, A. Peterman and E. de Rafael, Recent developments in the comparison between theory and experiments in quantum electrodynamics, Phys. Rept. 3 (1972) 193 [INSPIRE]. 
[17] G. Abbiendi et al., Measuring the leading hadronic contribution to the muon $g-2$ via $\mu$ e scattering, Eur. Phys. J. C 77 (2017) 139 [arXiv:1609.08987] [INSPIRE].

[18] A.B. Arbuzov, D. Haidt, C. Matteuzzi, M. Paganoni and L. Trentadue, The Running of the electromagnetic coupling $\alpha$ in small angle Bhabha scattering, Eur. Phys. J. C 34 (2004) 267 [hep-ph/0402211] [INSPIRE].

[19] OPAL collaboration, Measurement of the running of the QED coupling in small-angle Bhabha scattering at LEP, Eur. Phys. J. C 45 (2006) 1 [hep-ex/0505072] [inSPIRE].

[20] A.I. Nikishov, Radiative corrections to the scattering of $\mu$ mesons on electrons, Sov. Phys. JETP 12 (1961) 529 [INSPIRE].

[21] K.E. Eriksson, Radiative corrections to muon-electron scattering, Nuovo Cimento 19 (1961) 1029.

[22] K.E. Eriksson, B. Larsson and G.A. Rinander, Radiative corrections to muon-electron scattering, Nuovo Cimento 30 (1963) 1434.

[23] P. Van Nieuwenhuizen, Muon-electron scattering cross section to order $\alpha^{3}$, Nucl. Phys. B 28 (1971) 429 [INSPIRE].

[24] G. D'Ambrosio, Electron Muon Scattering in the Electroweak Unified Theory, Lett. Nuovo Cim. 38 (1983) 593 [INSPIRE].

[25] T.V. Kukhto, N.M. Shumeiko and S.I. Timoshin, Radiative Corrections in Polarized Electron Muon Elastic Scattering, J. Phys. G 13 (1987) 725 [inSPIRE].

[26] D.Y. Bardin and L. Kalinovskaya, QED corrections for polarized elastic $\mu$ e scattering, hep-ph/9712310 [INSPIRE].

[27] N. Kaiser, Radiative corrections to lepton-lepton scattering revisited, J. Phys. G 37 (2010) 115005 [INSPIRE].

[28] P. Mastrolia, M. Passera, A. Primo and U. Schubert, Master integrals for the NNLO virtual corrections to $\mu$ e scattering in QED: the planar graphs, JHEP 11 (2017) 198 [arXiv: 1709.07435] [INSPIRE].

[29] S. Di Vita, S. Laporta, P. Mastrolia, A. Primo and U. Schubert, Master integrals for the $N N L O$ virtual corrections to $\mu$ scattering in QED: the non-planar graphs, JHEP 09 (2018) 016 [arXiv: 1806.08241] [INSPIRE].

[30] M. Fael, Hadronic corrections to $\mu$-e scattering at NNLO with space-like data, arXiv: 1808.08233 [INSPIRE].

[31] M. Fael and M. Passera, Muon-electron scattering at NNLO: the hadronic corrections, arXiv: 1901.03106 [INSPIRE].

[32] M. Bohm, A. Denner and H. Joos, Gauge theories of the strong and electroweak interaction, Teubner, Stuttgart Germany (2001).

[33] M. Steinhauser, Leptonic contribution to the effective electromagnetic coupling constant up to three loops, Phys. Lett. B 429 (1998) 158 [hep-ph/9803313] [InSPIRE].

[34] C. Sturm, Leptonic contributions to the effective electromagnetic coupling at four-loop order in QED, Nucl. Phys. B 874 (2013) 698 [arXiv:1305.0581] [INSPIRE].

[35] G. Degrassi and A. Vicini, Two loop renormalization of the electric charge in the standard model, Phys. Rev. D 69 (2004) 073007 [hep-ph/0307122] [InSPIRE]. 
[36] F. Jegerlehner, Variations on Photon Vacuum Polarization, arXiv:1711.06089 [INSPIRE].

[37] B. Ruijl, T. Ueda and J. Vermaseren, FORM version 4.2, arXiv:1707.06453 [INSPIRE].

[38] J. Kuipers, T. Ueda, J.A.M. Vermaseren and J. Vollinga, FORM version 4.0, Comput. Phys. Commun. 184 (2013) 1453 [arXiv: 1203.6543] [INSPIRE].

[39] T. Hahn, Feynman Diagram Calculations with FeynArts, FormCalc and Loop Tools, PoS (ACAT2010) 078 (2010) [arXiv: 1006.2231] [INSPIRE].

[40] T. Hahn and M. Pérez-Victoria, Automatized one loop calculations in four-dimensions and D-dimensions, Comput. Phys. Commun. 118 (1999) 153 [hep-ph/9807565] [InSPIRE].

[41] A. Denner, S. Dittmaier and L. Hofer, COLLIER: a fortran-based Complex One-Loop LIbrary in Extended Regularizations, Comput. Phys. Commun. 212 (2017) 220 [arXiv: 1604.06792] [INSPIRE].

[42] S. Actis, A. Denner, L. Hofer, J.-N. Lang, A. Scharf and S. Uccirati, RECOLA: REcursive Computation of One-Loop Amplitudes, Comput. Phys. Commun. 214 (2017) 140 [arXiv: 1605.01090] [INSPIRE].

[43] G. 't Hooft and M.J.G. Veltman, Scalar One Loop Integrals, Nucl. Phys. B 153 (1979) 365 [INSPIRE].

[44] W. Beenakker and A. Denner, Infrared Divergent Scalar Box Integrals With Applications in the Electroweak Standard Model, Nucl. Phys. B 338 (1990) 349 [INSPIRE].

[45] S. Dittmaier, Separation of soft and collinear singularities from one loop $N$ point integrals, Nucl. Phys. B 675 (2003) 447 [hep-ph/0308246] [INSPIRE].

[46] A. Denner and S. Dittmaier, Scalar one-loop 4-point integrals, Nucl. Phys. B 844 (2011) 199 [arXiv: 1005.2076] [INSPIRE].

[47] G. Passarino and M.J.G. Veltman, One Loop Corrections for $e^{+} e^{-}$Annihilation Into $\mu^{+} \mu^{-}$ in the Weinberg Model, Nucl. Phys. B 160 (1979) 151 [INSPIRE].

[48] A. Denner and S. Dittmaier, Reduction of one loop tensor five point integrals, Nucl. Phys. B 658 (2003) 175 [hep-ph/0212259] [INSPIRE].

[49] A. Denner and S. Dittmaier, Reduction schemes for one-loop tensor integrals, Nucl. Phys. B 734 (2006) 62 [hep-ph/0509141] [INSPIRE].

[50] T. Engel, C. Gnendiger, A. Signer and Y. Ulrich, Small-mass effects in heavy-to-light form factors, arXiv: 1811.06461 [INSPIRE].

[51] S. Alioli et al., Precision studies of observables in $p p \rightarrow W \rightarrow l \nu_{l}$ and $p p \rightarrow \gamma, Z \rightarrow l^{+} l^{-}$ processes at the LHC, Eur. Phys. J. C 77 (2017) 280 [arXiv:1606.02330] [InSPIRE].

[52] M. Fael and M. Passera, private communication (2018).

[53] NA7 collaboration, A Measurement of the Space-Like Pion Electromagnetic Form Factor, Nucl. Phys. B 277 (1986) 168 [inSPIRE].

[54] G. Balossini, C.M. Carloni Calame, G. Montagna, O. Nicrosini and F. Piccinini, Matching perturbative and parton shower corrections to Bhabha process at flavour factories, Nucl. Phys. B 758 (2006) 227 [hep-ph/0607181] [INSPIRE].

[55] G. Balossini, C. Bignamini, C.M. Carloni Calame, G. Montagna, O. Nicrosini and F. Piccinini, Photon pair production at flavour factories with per mille accuracy, Phys. Lett. B 663 (2008) 209 [arXiv:0801.3360] [INSPIRE]. 
[56] Working Group on Radiative Corrections and Monte Carlo Generators for LOW ENERGIES collaboration, Quest for precision in hadronic cross sections at low energy: Monte Carlo tools vs. experimental data, Eur. Phys. J. C 66 (2010) 585 [arXiv:0912.0749] [INSPIRE].

[57] C.M. Carloni Calame, G. Montagna, O. Nicrosini and A. Vicini, Precision electroweak calculation of the charged current Drell-Yan process, JHEP 12 (2006) 016 [hep-ph/0609170] [INSPIRE].

[58] C.M. Carloni Calame, G. Montagna, O. Nicrosini and A. Vicini, Precision electroweak calculation of the production of a high transverse-momentum lepton pair at hadron colliders, JHEP 10 (2007) 109 [arXiv:0710.1722] [INSPIRE].

[59] S. Boselli, C.M. Carloni Calame, G. Montagna, O. Nicrosini and F. Piccinini, Higgs boson decay into four leptons at NLOPS electroweak accuracy, JHEP 06 (2015) 023 [arXiv: 1503.07394] [INSPIRE]. 